\title{
Coordinated and controlled mobility of multiple sinks for maximizing the lifetime of wireless sensor networks
}

\author{
Stefano Basagni • Alessio Carosi • Chiara Petrioli • \\ Cynthia A. Phillips
}

Published online: 8 January 2011

(C) Springer Science+Business Media, LLC 2011

\begin{abstract}
We define scalable models and distributed heuristics for the concurrent and coordinated movement of multiple sinks in a wireless sensor network, a case that presents significant challenges compared to the widely investigated case of a single mobile sink. Our objective is that of maximizing the network lifetime defined as the time from the start of network operations till the failure of the first node. We contribute to this problem providing three new results. We first define a linear program (LP) whose solution provides a provable upper bound on the maximum lifetime possible for any given number of sinks. We then develop a centralized heuristic that runs in polynomial time given the solution to the LP. We also define a deployable distributed heuristic for coordinating the motion of multiple sinks through the network. We demonstrate the performance of the proposed heuristics via ns2-based simulations. The observed results show that our distributed heuristic achieves network lifetimes that are remarkably close to the optimum ones, resulting also in significant improvements over the cases of deploying the sinks
\end{abstract}

\section{S. Basagni}

Department of Electrical and Computer Engineering,

Northeastern University, Boston, MA, USA

e-mail: basagni@ece.neu.edu

A. Carosi - C. Petrioli $(\bowtie)$

Dipartimento di Informatica, Università di Roma "La Sapienza",

Rome, Italy

e-mail: petrioli@di.uniroma1.it

A. Carosi

e-mail: carosi@di.uniroma1.it

C. A. Phillips

Sandia National Laboratories, Albuquerque, NM, USA

e-mail: caphill@sandia.gov statically, of random sink mobility and of heuristics previously proposed for restricted sink movements.

Keywords Wireless sensor networks . Mobility management $\cdot$ Sink mobility . Multi-sink mobile sensor networks

\section{Introduction}

One of the most promising recent research directions for performance improvement in wireless sensor networks (WSNs) [1] is exploiting the mobility of some of the network components. This mitigates the so called sink neighborhood problem, where many nodes send packets to a few data collection points (the sinks), funneling data through the network and placing increasing burdens on sensors close to the sinks. If the sinks are statically deployed, sensors near the sink expend energy at a rate much faster than sensors far from the sinks. When these nodes have drained their batteries, the sink cannot receive any further packets. Moving network componentswhether the sensors or the sinks - can better balance energy depletion among the nodes, prolonging network lifetime, i.e., the time the network is able to perform its functions. ${ }^{1}$ Devices could move independently of the network status.

\footnotetext{
${ }^{1}$ Definitions of network lifetime vary, and depend on applications. In this paper we adopt the widely used definition introduced in [2] and used in many works (see [3, 4] and references therein) where lifetime is defined as the time until the failure (here for energy depletion) of the first node. This definition may seem too pessimistic, and many advocate definitions based on the failure of a certain percentage of the nodes. However, robust, energy-efficient protocols for WSNs tend to balance energy consumption among the nodes. So when the first node dies, many other nodes are also about to die.
} 
For instance, they can travel randomly through the nodes, or in predetermined oblivious patterns (such as snaking or in a spiral). Alternatively, the devices could choose to move to specific locations based on key network parameters, such as the energy available at the nodes, energy consumption patterns, traffic load, etc. This is called controlled mobility.

In this paper we investigate network performance improvement via controlled and coordinated sink mobility. Appropriately scheduling sink movements can enable applications where long network lifetime is critical. For instance, structural health monitoring, as of a subway tunnel, is naturally performed by embedding small sensors into the structure. The sensors transmit data to small portable computers (sinks) which have high data rate connections with the monitoring center. Moving these sinks over time based upon evolving network conditions remarkably prolongs sensor lifetime without seriously impacting other metrics, as we show in this paper.

This paper provides clear evidence that controlled and coordinated mobility of multiple sinks achieves significantly better performance than uncontrolled movement or static sinks. Our review of the initial research on this topic (presented in details in the next section) found no analytical solutions for controlled and coordinated mobility of multiple sinks. We have also found no distributed protocols for coordinated and controlled mobility of sinks, which are needed for realistic deployments. We begin to fill this gap by making a threefold contribution. We provide a new scalable mathematical model that provides a provable upper bound on network lifetime. We give a centralized heuristic to compute sink movement schedules. These solutions are needed to provide provable bounds on the best possible network performance (e.g., the best achievable lifetime) as well as for benchmarking more realistic protocols. The third contribution is a realistically deployable distributed heuristic for the coordinated and controlled movement of multiple sinks.

Our new mathematical model flexibly captures realistic aspects of WSNs. It allows varying sensor deployment and has several parameters to control data generation rate, routing schemes, and energy costs for sensor tasks. The mixed integer linear programming (MILP) model defined for a single mobile sink [5] cannot be extended directly to multiple sinks. The main challenge here is scaling to model networks with hundreds of sensors or more. This is because at any given time a suitable MILP model must choose an active sink to receive the packets from each sensor. Natural linear ways to propagate this choice for calculating energy consumption yield weak linear programming (LP) relaxations and therefore long solve times. We achieve scalability by defining an LP model that provides an upper bound on the maximum lifetime possible for any given number of sinks. The LP has an exponential number of constraints, but we can compute the optimal solution efficiently by iteratively generating and adding a violated constraint and re-solving the LP a polynomial number of times. Our solution is quite technical, but allows us to compute an upper bound on the maximum lifetime for WSNs that are quite realistic, e.g., made up of 400 nodes, with 64 different positions where the sinks can sojourn (called sink sites), and with 5 sinks concurrently roaming throughout the network.

Starting from the output of the LP we define a new polynomial-time centralized heuristic for finding a full schedule for the movement of the sinks. Solutions provided by our scalable heuristic (described in detail in Sect. 4) obtain network lifetimes that are less than $1 \%$ below the upper bound obtained from the LP-based relaxation.

A further contribution of this work is the definition of a realistically deployable distributed protocol for controlling and coordinating the motion of multiple sinks through the network. The idea behind our protocol is to trigger the movement of each sink depending on the expected lifetime improvement that can be obtained by that move. Movements are dictated by the nodes' residual energy and by energy consumption patterns. A sink moves if and only if sojourning at a new position yields a longer (expected) lifetime (controlled sink mobility). By gathering network state information efficiently, sinks can make this decision locally and share it with fellow sinks. So, sink decisions take into account the decisions of others (sink coordination).

We conclude the paper by showing the results of a comparative performance evaluation of the proposed solutions, namely, the LP-based upper bound, the centralized heuristic, the distributed protocols for sink mobility, and protocols where sink mobility is uncontrolled and random. We also compare all these mobility schemes to the case where the sinks are statically and optimally placed and against the best performing solutions for multiple sink mobility presented by Azad and Chockalingam [6]. The experimental results show that our distributed heuristic achieves network lifetimes that are remarkably close to optimal. They were between 5.5 and $25.3 \%$ below the upper bound in all the scenarios we considered. The improvements over random sink mobility are also significant: up to $88.2 \%$. The lifetime from our distributed algorithm can be fourfold higher than the lifetime from optimally placed static sinks and up to $40 \%$ higher than the lifetime from Azad and Chockalingam's heuristics Max-Min-RE and MinDiff-RE [6]. These results show clearly that controlling and coordinating the mobility of sinks remarkably prolongs the lifetime of realistically deployable WSNs.

The rest of the paper is organized as follows. In the next section we review previous works on the placement and 
exploitation of mobility of multiple sinks. We give problem formulation details in Sect. 3. In Sect. 4 we describe the LP model that provides upper bounds on the optimal network lifetime. The scalable centralized heuristic and the distributed heuristic are described in Sects. 5 and 6, respectively. In Sect. 7 we present ns2-based simulations to validate our centralized and distributed schemes and to compare them to the upper bound, the Max-Min-RE and MinDiff-RE centralized heuristics, static sink placement and random mobility. Section 8 concludes the paper.

\section{Related works}

Improving WSN lifetime by exploiting the mobility of multiple sinks involves determining routes for the sinks and their sojourn times at designated sites so that the lifetime is maximized.

Gandham et al. [7] propose the first work on multi-sink WSNs, improving lifetime indirectly by greedily minimizing nodal energy consumption. They divide time into rounds. At the beginning of each round they solve a static sink placement problem to reposition the sinks based on the current energy in the network nodes. Specifically, they centrally gather the residual energy of all nodes. Then they solve an integer linear program (ILP) to determine new sink locations that minimize the maximum energy expended by any node in the next round, subject to the constraint that no node expends more than an $\alpha$ fraction of its current residual energy. The ILP also determines packet transmission rates for each node to its neighbors. The sensors then route packets to outgoing edges in a round robin based on the proportions given by the ILP. Experiments show that this algorithm with three sinks extends lifetime considerably compared to deploying one static sink. This first effort to address deployment of multiple sinks does not consider many important characteristics of WSNs. For instance, although the ILP models minimize nodal energy consumption in each round, this greedy approach does not guarantee a globally optimal lifetime. Moreover, the ILP models do not consider energy spent for route management, which is non-negligible, and is routing dependent.

Azad and Chockalingam [6] propose three centralized heuristics for the models and setting of [7], where $s \geq 1$ sinks can sojourn only at $v \geq s$ sites on the boundary of the network deployment region. In the first heuristic, Top- $K_{\max }$, the authors let the residual energy of a sink site $i$ be the residual energy of the sensor closest to $i$. At the start of each round, they place the $s$ sinks at the $s$ sink sites with the highest residual energy. The second heuristic is called Max-Min-RE because it chooses a sink configuration where the most heavily loaded node (the one with minimum residual energy, Min-RE) will have the maximum energy at the end of the next round. To more evenly drain the nodes, the third heuristic (MinDiff-RE), chooses the configuration that minimizes (Min) the difference (Diff) between the maximum and minimum residual energy (RE) over all nodes. The latter two solutions require the enumeration of all possible $\left(\begin{array}{l}v \\ s\end{array}\right)$ sink placements, and are therefore viable only when the number of sinks is very limited.

Ren et al. [8] investigate the impact of multiple mobile sinks on end-to-end packet delay and energy consumption. They consider trade offs for optimizing both. Specifically, deploying sinks moving in an uncontrolled way (random way-point), they investigate the impact of sink number, speed, sink transmission radius, and data routing on performance. Single-hop collection minimizes the energy but results in higher latencies, so that in many applications multi-hop communication is the only viable option. The authors conclude that the number of sinks, their speed and the routing can be tuned to provide acceptable delays and energy consumption, i.e., to obtain a required network lifetime. They also observe through simulations that (uncontrolled) sink mobility yields more balanced energy consumption compared to static sinks. Chen and Ma, with $\mathrm{Yu}$, continue to investigate this topic in [9], where rather than mobile sinks, they explore a three-tier architecture where cellular phones, whose mobility is uncontrolled, act similarly to data MULEs and carry data to a single base station.

Recently, Chatzigiannakis et al. [10] propose three solutions for efficient data collection via mobile sinks. They wish to move sinks to reduce nodal energy consumption, prolong network lifetime, increase delivery rate, and decrease packet latency with respect to the case with one sink roaming through the network. Packet routing from the sensors to the sinks is single hop, i.e., a sensor must be visited by a sink in order to deliver its data. The first protocol is centralized: The deployment area is partitioned into equally sized regions, each of which is assigned a sink that traverses it exhaustively in a snake fashion. This solution is particularly suitable when the network is fairly stable and homogeneous. No actual coordination is needed among the sinks, since each region is an independent collection problem. The second protocol requires some loose coordination of the sinks. The $s$ sinks are initially deployed randomly. Each moves in a random walk through the entire network area. Each sink transmits a beacon message containing its ID, its position, speed and direction. This information is stored at the sensors that receive it for a predefined time $t$. If another sink comes by within $t$ time from the passing of a previous sink, the new one changes 
its direction, thus avoiding traversing an area visited recently by some other sink. The third protocol partitions the network into small clusters and assigns clusters to sinks. Clusters have weights that depend on critical cluster parameters such as the traffic load, nodal residual energy, etc. If there are $s$ sinks, the protocol then combines clusters into $s$ groups with roughly equal weight. Every sink does a random walk through its clusters, collecting data from nodes in its group in a single-hop fashion. Experimental results show that the proposed protocols achieve good performance using only up to four sinks.

\section{Problem formulation}

We consider networks with a small number $s$ of mobile sinks that collect data from a large set $N$ of deployed resourceconstrained sensor nodes. Sinks are allowed to sojourn at any of a finite number $v$ of designated sink sites from a set $V,|V|=v$. At any time, a sink is either at a sink site or it is moving. If it is at a sink site, it can be either active, i.e., available to receive sensor data, or inactive. ${ }^{2}$ A subset of $1 \leq k \leq s$ sites hosting active sinks is called a configuration, and the set of all possible sink configurations is denoted by $C$. A sink configuration changes whenever a sink becomes active or inactive. A moving sink is always inactive, and therefore it cannot receive packets. When a sink at a sink site becomes active, it must broadcast its availability to the sensors. Similarly, when it becomes inactive it must broadcast that it is not available anymore. These broadcasts consume energy from the sensors and are sink-site dependent. We require that at least one sink is active at all times. This design choice allows the sensors to always have a destination for their packets. This has the benefit of avoiding high packet end-to-end latencies because nodes do not need to buffer packets while the sinks are moving.

Each sensor $p \in N$ has initial energy ${ }^{3} e_{p}$. It generates data at a rate of $r_{p}$ packets per second. These packets are routed either directly or via a multi-hop path to a sink (e.g., the one sojourning at a closest site) according to a given routing protocol. Sensor $p$ requires $\alpha_{p}$ joules per packet for sensing, creating, and transmitting its own (i.e., locally generated) packets. It requires $\beta_{p}$ joules per packet for receiving and relaying a packet for another sensor.

\footnotetext{
$\overline{2}$ One might expect that an optimal schedule will use all stationary sinks at all times. However, there may be times when it is best not to use one even if it is available. This is because the extra sink will affect packet routes, possibly affecting energy balance negatively.

${ }^{3}$ If the protocol involves a training phase, i.e., a phase through which the sensors and the sinks learn about parameters needed to start protocol operations, $e_{p}$ is the energy after the training.
}

Let $0 \leq y_{p w c} \leq 1$ be the fraction of traffic that sensor $p \in N$ sends to a sink at site $w \in V$ when the sink configuration is $c \in C$. When sensor $p \in N$ is sending some traffic to sink site $w \in V$, let $0 \leq \rho_{p q w} \leq 1$ be the fraction of the $p$-to- $w$ traffic sent through sensor $q \in N$. Routing protocols proposed for WSNs can use multiple routes between a sensor and a sink. Our formulation allows arbitrarily complex routing strategies provided each sensor decides where to send data and how to route it based only on the sink configuration.

Our objective is to find a schedule of sink movements (i.e., a sequence of configurations) that maximizes the network lifetime.

Some of the configurations of a schedule are selected based on their energy efficiency and their ability, as a group, to balance energy depletion among the network nodes. These configurations are called major configurations. We want to stay in them for the time necessary to maximize the network lifetime. However, major configurations may not be enough to compose a schedule. This is because we require at least one active sink at all times and traveling sinks to have enough time to move from one site to the new selected one. Therefore, a schedule may need one or two intermediate configurations to make the transition between two major configurations. Those configurations used only to move between major configurations are called transient configurations.

Figure 1 illustrates the need for transient configurations in a scenario with six sinks. Moving from configuration $c_{i}$ to configuration $c_{i+1}$ by simply deactivating all sinks and letting them move to their new sites would not leave any sink active, as required. To go from $c_{i}$ to $c_{i+1}$, we use 2 transient configurations $c_{i}^{a}$ and $c_{i}^{b}$. From $c_{i}$, we let the sinks in the set $M_{1}$ (boxed in the figure) move, while those in sites $v_{1}, v_{3}$ and $v_{5}$ stay active. Thus $c_{i}^{a}=\left\{v_{1}, v_{3}, v_{5}\right\}$. Moving from $c_{i}^{a}$ to $c_{i}^{b}$, the sinks that left sites in $M_{1}$ arrive at their new locations in $c_{i}^{b}=\left\{v_{7}, v_{9}, v_{11}\right\}$ and simultaneously, those in $c_{i}^{a}$ deactivate to move. When sinks from sites in $c_{i}^{a}$ arrive at their new locations in $c_{i+1}-c_{i}^{b}=\left\{v_{8}, v_{10}, v_{12}\right\}$, they activate to put the system into configuration $c_{i+1}$. In "Appendix" we show that at most two transient configurations are needed to move between any pair of configurations.

Consider a sequence $\mathcal{C}=c_{1}, c_{2}, \ldots, c_{L}$ of configurations, either major or transient, that provide a schedule of sink movements that solve our problem. Configuration $c_{i}$ must hold for the time to broadcast the sink site (de)activations necessary to move from $c_{i}$ into $c_{i+1}$ plus the time required to move sinks to new locations for $c_{i+1}$. For a given sequence $\mathcal{C}$, we denote this minimum time for configuration $c_{i}$ as $\tau^{\mathcal{C}}\left(c_{i}\right)$. We also require that major configurations hold for at least a fixed time threshold 


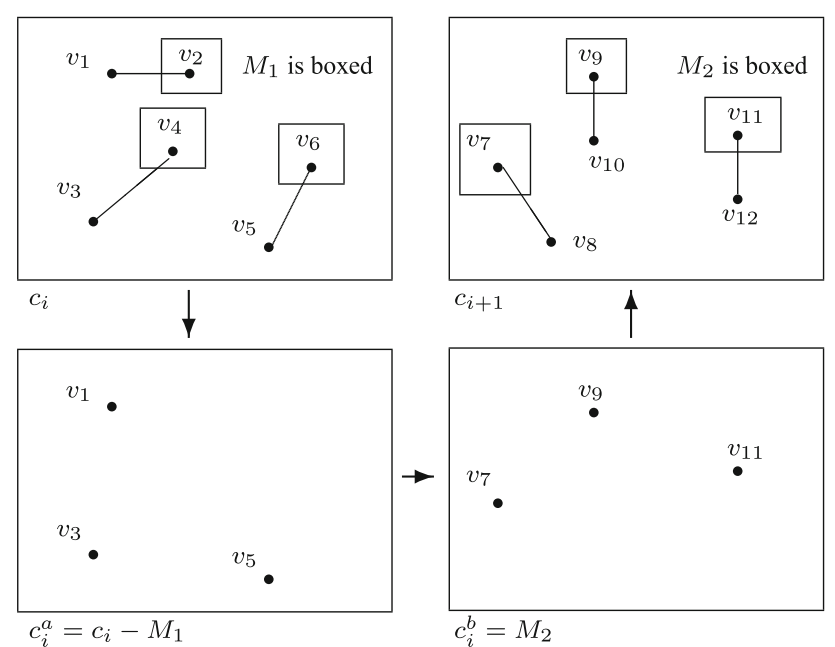

Fig. 1 Illustration of calculating a two-step transition between major configurations $c_{i}$ and $c_{i+1}$. The sinks in set $M_{1}$ (one element from each matched pair in $c_{i}$ ) deactivate and move while the remaining sinks stay in configuration $c_{i}^{a}$. The sinks originally in $M_{1}$ appear in configuration $c_{i}^{b}$ at locations $M_{2}$, which represent one element from each matched pair in the new configuration $c_{i+1}$

$t_{\min } \gg \max _{i} \tau^{\mathcal{C}}\left(c_{i}\right)$ seconds. ${ }^{4}$ By varying the length of $t_{\min }$ we can explore trade-offs between sink mobility and network lifetime.

Our problem is formally defined as follows.

Problem formulation. Determine an ordered set $\mathcal{C}_{m}=$ $\left\{c_{0}, c_{1}, \ldots, c_{\ell}\right\}$ of major configurations and a time $t_{i} \geq t_{\min }$ for each selected configuration $c_{i}, i=0, \ldots, \ell$. Also determine transient configurations $\mathcal{C}_{t}=\left\{c_{0}^{a}, c_{0}^{b}, c_{1}^{a}, c_{1}^{b}, \ldots\right.$, $\left.c_{\ell-1}^{a}, c_{\ell-1}^{b}\right\}$ and times $t_{i}^{a}, t_{i}^{b}$ for $i=0, \ldots, \ell-1$. The ordered pair of transient configurations $\left(c_{i}^{a}, c_{i}^{b}\right)$ defines a most direct transition between major configurations $c_{i}$ and $c_{i+1}$. Call the sequence of major configurations with appropriately interleaved transient configurations $\mathcal{C}$. If a transient configuration $c$ exists, then it differs from the preceding configuration and lasts at least $\tau^{\mathcal{C}}(c)$ seconds. If one or both of the transient configurations do not exist, the configuration lasts zero seconds and is equal to the configuration preceding it. By convention, $t_{i}^{a} \geq t_{i}^{b}$, so the " $b$ " configuration exists only if moving between configurations $c_{i}$ and $c_{i+1}$ requires two transient configurations. For convenience, let $t_{\ell}^{a}=t_{\ell}^{b}=0$. We wish to maximize the network lifetime $\Lambda=\sum_{i \in 0 \ldots \ell}\left(t_{i}+t_{i}^{a}+t_{i}^{b}\right)$.

Our definition of network lifetime requires every sensor to have enough initial energy to support all the selected configurations for the selected amount of time. This means that the energy paid by each node $p$ for generating, transmitting, receiving and relaying packets while in each of the

\footnotetext{
${ }^{4}$ Holding major configurations for a longer time allows routes to stabilize and run for enough time to justify the cost of changing the configuration.
}

selected configurations $\mathcal{C}_{m} \cup \mathcal{C}_{t}$ plus the energy spent by the node for route maintenance operations associated with configuration changes does not exceed the nodal initial energy $e_{p}$. (Our formulation accounts for all the energy costs incurring at the network nodes during the network operations.) After $\Lambda$ seconds, at least one sensor dies.

\section{A mathematical model for sink mobility}

In this section we describe an LP whose solution provides an upper bound on the maximum lifetime of a sensor network with $s$ mobile sinks. The LP is a relaxation of the mobile sinks problem. It selects a set of (major) configurations and the time for each configuration. It ignores the ordering of configurations and the transitions between them, minimum times for configurations to hold, and energy costs for broadcasting sink (de)activations. Since any feasible schedule for sink movements must obey these additional configuration constraints and the required broadcasts consume sensor energy, the lifetime of a real schedule can be no longer than the lifetime of the optimal LP solution.

The LP has only one type of variable. Let $t_{c}$ be the time configuration $c \in C$ holds. There are $\sum_{k=1}^{s}\left(\begin{array}{l}v \\ k\end{array}\right)$ ways to place at most $s$ sinks on $v$ sites, which is exponential in $s$. However, we can solve this LP while explicitly including the $t_{c}$ variables for only a polynomial number of configurations $c$. The rest are implicitly 0 .

The LP model is:

$\operatorname{maximize} \sum_{c \in C} t_{c}$

subject to:

$\sum_{c \in C}\left(\alpha_{p} r_{p} t_{c}+\sum_{q \neq p \in N, w \in V} r_{q} \beta_{p} \rho_{q p w} y_{q w c} t_{c}\right) \leq e_{p}, \forall p \in N$.

The objective function maximizes network lifetime. The constraints require all sensors to be alive throughout the network lifetime. Given a sensor $p \in N$ and a configuration $c \in C$, the expression in the parentheses on the left side of the constraint for sensor $p$ gives its energy consumption while the system is in configuration $c$. More specifically, $\alpha_{p} r_{p}$ is the energy consumption per unit time for sensing, creating and transmitting locally generated packets. Therefore, $\alpha_{p} r_{p} t_{c}$ is the total energy consumed by $p$ in configuration $c$ for packets it generates. The second term computes the energy that sensor $p$ expends routing packets for other sensors during configuration $c$. Each term in this sum depends on the data rate $r_{q}$ of sensor $q$, which might route through sensor $p$ depending on sensor $q$ 's choices of sink and choice(s) of route. Recall that parameter $y_{q w c}$ 
indicates the fraction of sensor $q$ 's traffic that goes to site $w$ in configuration $c$ (choice of sink). We have $y_{q w c}>0$ only if a sink sojourns at site $w$ in configuration $c$ and sensor $q$ sends packets to a sink at site $w$ in configuration $c$. Also recall parameter $\rho_{q p w}$ is the fraction of $q$ 's traffic to sink $w$ that passes through sensor $p$ (choice of route). Therefore, $r_{q} \beta_{p} \rho_{q p w} y_{q w c} t_{c}$ represents the energy consumed at node $p$ for relaying packets generated by $q$ during the time that configuration $c$ holds.

\subsection{Solving the LP}

We will use a general method for solving LPs that have an exponential number of constraints but a polynomial number of variables. Our LP has an exponential number of variables, but a polynomial number of constraints. So we will optimize our LP (the primal) by instead optimizing its dual [11]. This is another LP, derived mechanically from the primal, which switches the optimization direction (in this case to a minimization). Each constraint in the primal corresponds to a variable in the dual and each variable in the primal corresponds to a constraint in the dual. So the dual of our LP has an exponential number of constraints rather than an exponential number of variables. The objectives of the two LPs are equal at optimality. The dual of our LP is:

$\operatorname{minimize} \sum_{p \in N} e_{p} u_{p}$

subject to:

$$
\begin{gathered}
\sum_{p \in N} u_{p}\left(r_{p} \alpha_{p}+\sum_{q \neq p \in N} \sum_{w \in V} r_{q} \beta_{p} \rho_{q p w} y_{q w c}\right) \geq 1 \\
\forall c \in C, \\
u_{p} \geq 0 \forall p \in N .
\end{gathered}
$$

We can satisfy an exponential-size set of constraints by only explicitly enforcing a polynomial number of them provided we have a separation algorithm. Suppose we wish to enforce a set of constraints $\mathcal{X}$. A separation algorithm accepts a solution vector for the LP that only explicitly lists a subset of the constraints. It returns a constraint $x \in \mathcal{X}$ that is violated, or verifies all are satisfied. As a theoretical results, such a separation procedure, when combined with the ellipsoid algorithm, is guaranteed to converge to the optimal LP solution in a polynomial number of iterations. See [12-14] and especially [15, Chap. 14], for some of many discussions of this technique.

Although it has good theoretical bounds, the ellipsoid algorithm is not very fast. That is why linear programming solvers use simplex or barrier methods rather than the theoretically good ellipsoid algorithm for linear programming. Similarly, we use a different, simple approach for solving our linear program with separation. This method is typically much better than using ellipsoid in practice.

To use a separation algorithm to solve a large LP, we begin by explicitly listing a small (possibly empty) subset of the constraints in $\mathcal{X}$. We then solve the LP and pass the solution to the separation algorithm. If all the constraints are satisfied, we are done. The separation algorithm has given a computational proof that all the constraints are satisfied and thus all unlisted constraints are redundant at optimality. Otherwise, we add the violated constraint it returns, solve the new LP, and so on.

An efficient separation algorithm must exploit structure of the constraint family; it does not enumerate the constraints. Thus, a separation algorithm is customized for each problem, and there is no reason to believe a priori that this computation is tractable. We now describe how we can apply this approach to solving our dual LP.

Given a solution $u^{*}$ to the dual LP, we wish to find a most violated constraint, or determine that all are satisfied. One can separate by just finding any violated constraint, but finding a most violated constraint has better convergence properties. A violated constraint has the form

$\sum_{p \in N} u_{p}^{*}\left(r_{p} \alpha_{p}+\sum_{q \neq p \in N} \sum_{w \in V} r_{q} \beta_{p} \rho_{q p w} y_{q w c}\right)<1$

for some configuration $c$. A most violated constraint minimizes the value of

$\sum_{p \in N} u_{p}^{*} \sum_{q \neq p \in N} \sum_{w \in V} r_{q} \beta_{p} \rho_{q p w} y_{q w c}$

over all configurations $c$. If this value is strictly less than $1-\sum_{p \in N} u_{p}^{*} r_{p} \alpha_{p}$, then the constraint is violated and otherwise, all are satisfied.

Since we seek a configuration, the $y_{q w c}$ are now variables. We can drop the $c$ in the subscript, since we are computing a single configuration. Defining constant

$h_{q w}=r_{q} \sum_{p \neq q} u_{p}^{*} \beta_{p} \rho_{q p w}$,

our objective becomes minimize $\sum_{q \in N} \sum_{w \in V} h_{q w} y_{q w}$.

To find the optimal configuration, we must place up to $s$ sinks. We use a decision variable $x_{w}$, which is 1 if there is an active sink at site $w$ and 0 otherwise. As before, $y_{q w}>0$ if sensor $q$ sends to an active sink at site $w$ for the configuration we are building. Since there must be an active sink at site $w$ to receive traffic, we must ensure that $y_{q w}>0$ only if there is a sink at site $w$. The full separation formulation, which we call SEP, becomes:

$\operatorname{minimize} \sum_{q \in N} \sum_{w \in V} h_{q w} y_{q w}$

subject to: 
$\sum_{w \in V} x_{w} \leq s$

$\sum_{w \in V}^{w \in V} y_{q w}=1 \quad \forall q \in N$

$y_{q w} \leq x_{w} \quad \forall q \in N, w \in V$

$x_{i} \in\{0,1\}$.

The first constraint enforces the sink limit. The second set of constraints ensures that each sensor sends all its traffic to a set of sinks. The third set of constraints ensures that sensors send data only to a sink site that has an active sink. That is, if $x_{w}=0$, then $y_{q w}=0$ for all sensors $q$.

This MILP is a classic formulation for the $p$-median problem [16]. In the $p$-median problem, we wish to place $p$ facilities on any of $n$ locations. Each of $m$ customers must be served by an open facility, or a mix of open facilities. There is a service cost $c_{i j}$ for facility $i$ to serve customer $j$. If facility $i$ serves an $x_{i j}$ fraction of customer $j$ 's requirement, this costs $c_{i j} x_{i j}$. The goal is to minimize the total service cost. For our separation algorithm $p=s, n=|V|$, and $m=|N|$. This separation algorithm finds the configuration with the minimum total energy drain (summed over all sensors) when the energy cost is scaled by $u_{p}^{*}$.

The $p$-median problem is NP-complete. However, there are exact algorithms based on math-programming formulations that can effectively solve problems with ten thousand or more nodes and hundreds of thousands of customers [17]. This is well within or beyond the range of current sensor network sizes. Thus, although we cannot solve the LP in guaranteed polynomial time, we can solve it in practice for the sizes of LP we require, i.e., we can compute an upper bound on WSN lifetime.

The method above works for any possible assignment of sensors to the sinks to which they send their data. For instance, the network owner may decide to have the sensors send their packets to the closest sink, may forbid a source to send to a given sink due to latency constraints, or may list the preferred sinks according to a given ranking.

For sensor $q$ and sink site $w$, let $R_{q w}$ be the set of sink sites $u \neq w$ that are lower priority choices for sensor $q$ than site $w$. For example, if we must ensure that sensor $q$ sends only to a closest active sink, we can let $d(q, w)$ be the minimum distance (in hops) from sensor $q$ to sink site $w$. Then $R_{q w}$ includes the set of sink sites $u$ such that $d(q, u)>d(q, w)$, i.e., those that are farther away from sensor $q$ than site $w$ is. If there are ties for closest sink site, we can let the LP choose the best way to divide traffic among those sinks for a particular configuration. Alternatively, we can extend the $R_{q w}$ sets to enforce a total order by including sites $u$ such that $d(q, u)=d(q, w)$ where $w$ has a higher ID than $u$ ( $u$ loses the tie breaker with $w$ ). In order to enforce this sink selection strategy we add the following constraint to the SEP mixed-integer program: $\sum_{u \in R_{q w}} y_{q u} \leq 1-x_{w} \quad \forall q \in N, w \in V$.

This set of constraints enforces priority, for example to require sensor $q$ to send to a closest sink. If there is an active sink at site $w$ (i.e., $x_{w}=1$ ), then $q$ cannot send to any sink in $R_{q w}$. Thus all the sink selection variables corresponding to sites in $R_{q w}$ will be 0 . Adding the constraints to select a closest open facility preferentially leads to a more constrained $p$-median problem. Fortunately, this slightly-modified version appears to be quite tractable for our data sets.

We can also enforce any specific set of $y_{q w c}$ choices for a tractably-sized set of configurations $c \in C$. Suppose the separation algorithm returns a configuration $c$ with choices of $y_{q w c}$ that do not match those given by the network owner. We first check to see if the constraint is still violated with the correct $y_{q w c}$ parameters. If so, we add the new constraint using the correct $y$ values. If it is not violated, we will try again, forbidding the separation MILP from returning the same precise set of sinks. Suppose configuration $c$ has the $s$ sinks in locations $w_{1}, w_{2}, \ldots, w_{s}$. To forbid the SEP integer program from returning the same configuration, add the following constraint:

$\sum_{i=1}^{s} x_{w_{i}} \leq s-1$.

This means at least one of the $s$ variables representing this set of sink sites must be 0 (not selected). This mechanism could for instance be exploited to forbid configurations that may overload some of the sinks.

\section{Centralized heuristic}

In this section, we describe a centralized heuristic that begins with the solution to the LP described in Sect. 4 and finds a feasible schedule for sink movements.

The heuristic chooses a subset of the configurations chosen by the LP as major configurations. It must order these major configurations and compute transitions between neighboring major configurations. The centralized heuristic must also determine the time each configuration holds such that all sensors have enough energy to serve all the configurations. Major configurations must hold for at least $t_{\text {min }}$ seconds and transient configurations must hold long enough to complete the required broadcasts and sink movements. The high-level operations of the centralized heuristic are as follows:

1. Solve the LP to obtain a solution $t_{c}^{*}$. Let $B=t_{\min }$.

2. Let $\mathcal{C} \subseteq C=\left\{c \in C \mid t_{c}^{*} \geq B\right\}$. That is, we select the set of configurations for which the LP assigns a time of at least $B$ (initially $t_{\mathrm{min}}$ ). 
3. Order the configurations in $\mathcal{C}$, preferably with adjacent configurations sharing some common sink sites. (This decreases the number of transient configurations that need to be added.)

4. Compute transient configurations between each pair of adjacent configurations.

5. Solve another final LP (LPF) to adjust the times for each configuration, enforce minimum times on configurations, and account for sink-movement broadcast costs.

6. If LPF is infeasible, increase $B$ (e.g., to drop the configuration(s) with the shortest time from $\mathcal{C}$ ) and return to step 2 .

We now consider each step of the centralized algorithm, starting with step 3 . We use a simple traveling salesperson (TSP) model on a graph where vertices represent the major configurations from the LP and where there is an edge between each pair of vertices. The edge $\left(c_{i}, c_{j}\right)$ is weighted by 1 plus the minimum number of transitions needed to move from $c_{i}$ to $c_{j}$ or vice versa, since this relationship is symmetric. The weights are 1,2 , or 3 given that at most two transient configurations are needed to go from a major configuration to the following one. ${ }^{5} \mathrm{We}$ wish to find a traveling salesman path (not a closed tour) among the chosen configurations. The optimal TSP minimizes the number of configurations we must add. These are tiny and easy problems for the free TSP code Concorde [18]. If we would like the heuristic to run in guaranteed polynomial time, we can approximate the optimal path using Christofides' heuristic for the symmetric TSP [19].

We now give a high-level description of how to compute the transient configuration(s) between two major configurations in step 4. If there are no intermediate transitions required, then we are done. If there is one intermediate transition, then this usually is the (non-empty) intersection of sites in the two major configurations. When the transition requires two transient configurations, there are many possible choices for the transient configurations. The sink movement schedule has the form $c_{i}, M \subset c_{i}, L \subset c_{i+1}, c_{i+1}$, where $c_{i}$ and $c_{i+1}$ are adjacent major configurations after step 3. We must choose a set of sinks to move first, leaving the remainder $(M)$ behind to receive messages, and compute which sites in the new configuration this set will occupy $(L)$. The way $L$ and $M$ are chosen ensures that transient configurations induce an energy consumption pattern similar to that of $c_{i}$ and $c_{i+1}$. In this way, as a whole, transient configurations show the same energy balancing properties of major configurations. Details are given in "Appendix".

\footnotetext{
5 Note that these weights are a metric. Weighting only by the number of transient states does not satisfy the triangle inequality.
}

We now consider the final LP. Because we have selected the precise set of configurations (steps 2-4), we now no longer have to allow for zero values of $t_{c}$. So we can enforce minimum times for configurations. Because we know the order of the configurations, we can account for route maintenance costs. Specifically, each sink in the initial configuration $c_{0}$ must broadcast its activation. Moving between $c_{i}$ and $c_{i+1}$, all sink sites in $c_{i}-c_{i+1}$ must broadcast their deactivation and all sinks in $c_{i+1}-c_{i}$ must broadcast their activation. Each broadcast from a sink site $w \in V$ has a potentially different energy cost for each sensor. Let $\gamma_{p}$ be the total energy cost for sensor $p$ for all route maintenance operations associated with the specific sequence of configurations. This is a constant (a function of the predetermined set of activations and deactivations). Thus $e_{p}-\gamma_{p}$ is the energy sensor $p$ has remaining for handling packets during the network lifetime. Let $\mathcal{C}_{m}$ be the last set of major configurations selected in step 2 , let $\mathcal{C}_{t}$ be the set of transient configurations computed in step 4 , and let $\mathcal{C}$ be the appropriately interleaved sequence of $\mathcal{C}_{m}$ and $\mathcal{C}_{t}$ Then the final LP, called LPF, is as follows:

$\operatorname{maximize} \sum_{c \in \mathcal{C}_{m} \cup \mathcal{C}_{t}} t_{c}$

subject to:

$$
\begin{gathered}
\sum_{c \in \mathcal{C}_{m} \cup \mathcal{C}_{t}}\left(\alpha_{p} r_{p} t_{c}+\sum_{q \in N, w \in V} r_{q} \beta_{p} \rho_{q p w} y_{q w c} t_{c}\right) \leq e_{p}-\gamma_{p} \\
\forall p \in N \\
t_{c} \geq t_{\min } \quad \forall c \in \mathcal{C}_{m} \\
t_{c} \geq \tau^{\mathcal{C}}(c) \quad \forall c \in \mathcal{C}_{t} .
\end{gathered}
$$

This LP has a polynomial number of configurations so we can solve it directly.

\section{Distributed heuristics}

In this section we introduce three distributed heuristics for sink mobility. The first one takes into account the nodal residual energy for deciding where to move the sinks. The second and third instead move each sink randomly and uniformly through the available sites. (The latter represent protocols where mobility is uncontrolled and uncoordinated. We will use them in the next section for benchmarking.)

\subsection{Controlled and coordinated sink mobility}

The distributed heuristic for sink mobility, called DIS, starts by placing $s>1$ sinks at any $s$ of the $v$ sites (initial configuration). The sinks then broadcast a packet to the nodes advertising their current position. Upon receiving 
this packet, nodes set up routes to their preferred sink according to a selected routing protocol. Each sink maintains estimates of the state of the network, such as energy level of the sensors, current traffic from the nodes sending to it, sink locations and travel information. Based on this information each sink guesses the network lifetime if all sinks remain in their current positions till the network dies.

Periodically (i.e., every $t_{\min }$ seconds) each sink decides whether to move or not. Based on its current estimates of the network information, it computes the expected change in lifetime if it were to move to another site not currently occupied or about to be occupied by another sink. If sites exist such that moving to one of those sites would extend the network lifetime more than $\delta t_{\min }$ seconds, the sink performs the following operations: It chooses the new site which induces the maximum expected network lifetime; It communicates to all the other sinks that it has decided to move to the selected new site; It tells the sensors currently reporting to it that it is on the move, shutting down the routes to its current site (sink deactivation), and moves to the new site. Upon arriving at the new site the sink broadcasts a packet advertising its new position thereby triggering route construction from the nodes for which it is the preferred sink (sink activation).

The sinks are aware of each other's status. Therefore a sink knows if all the other sinks are traveling. If a sink is the last active sink, it will wait for an activation message before moving. This ensures that there is always a sink to receive packets, which enables low latencies.

A sink $f$ 's periodic decision about whether to move or not depends on information about the state of the network. In order to determine whether the network lifetime will be longer if it moves, $f$ needs to know the nodal residual energy, the nodal data rate and the energy needed to communicate packets in the new configuration. Sink $f$ learns this information either by collecting it from its nodes, or by receiving it from fellow sinks. Sinks collect information about current nodal energy in the following way. The energy is divided into a constant number of levels. When the energy of a node decreases from a level to a lower one, a node piggybacks this information to a data packet. A sink also gathers information about its nodes' data rate by counting packets. Sinks periodically share this information with the other sinks. The energy costs incurred by a node in the new configuration are based on knowing which node will transmit to which sink in the new configuration, and on the energy cost associated with these transmissions. The first information depends on the rank of a given sink site in the priority list of a sender node, and the second on estimates of the $\rho_{q p w}$ (as defined in Sect. 4). Both priority lists and $\rho_{q p w}$ estimates are obtained at network set up, during a training phase. This phase can be performed by one sink, which can then share the information with all the others. The sink travels to each sink site and broadcasts a packet to make the sensor nodes aware of its current location. Upon receiving this packet the sensor nodes transmit test packets to the sink according to the routing protocol in use. Each test packet carries information about the route followed from its source to the current location of the sink. Each traversed hop is associated with the energy necessary to forward the packet through it. After receiving a few test packets from a node $q$, a sink sojourning at site $w$ is able to estimate, for each node $p$, the fraction of packets generated by $q$ that will be relayed by $p$ when $q$ transmits to a sink at that site (i.e., the sink is able to estimate $\rho_{q p w}$ ). A sink at $w$ knows the energy consumption needed by node $p$ to relay packets from $q$. During the training phase, sensor nodes also decide how good a sink site is for them, and return this information to the sink in the test packets. Therefore, a node creates a priority list of sink sites.

\subsection{Random mobility: RND and ZRND}

We define two sink mobility scheme intended to represent uncontrolled mobility.

The first scheme, called RND (for random scheme), works as follows. Every $t_{\min }$ seconds, a sink randomly and uniformly decides the next site to visit among all unoccupied sites and its current site. If the sink moves to a new site, it communicates its decision to its peers, so that no two sinks go to the same site. Sensor-to-sink multi-hop route management and sink movement coordination happens as for DIS.

In ZRND (for zone random scheme) instead, the deployment area is divided into $s$ zones, each associated to a given sink (and only to it, i.e., the zones are non-overlapping). Every $t_{\min }$ seconds the sink randomly and uniformly selects the next site among the ones in its own zone.

\section{Performance evaluation}

In this section we discuss the results of a simulation-based performance evaluation of the solutions proposed in this paper. The section is organized into three parts. We first introduce the simulators, the simulation scenarios and their parameters. Then, we compare the performance of our distributed heuristic DIS and of the centralized heuristic (CEN in the following) defined in Sect. 4 to the upper bound on the optimal network lifetime (OPT), and to lifetimes for random sink mobility (RND and ZRND) and for optimally placed static sinks (STATIC). Finally, we compare the performance of our solutions to the performance of the solutions for multiple sink mobility MaxMin-RE and MinDiff-RE presented in [6]. 


\subsection{Simulators and simulation scenarios}

The results for OPT have been obtained by solving the LP model defined in Sect. 4 with the commercial solver CPLEX [20] run on Linux-based 64bit dual-core computers. The various runs took from few hours to few days to produce results. We considered the two cases where the sensors send their data to the closest sink, or to the "best" sink, which is the one sending to whom maximizes network lifetime. The first choice comes as a natural one in the sense that is very easy to implement by all the heuristics, and it is the default one in our experiments.

We implemented the centralized heuristic CEN (Sect. 5) in a home-grown software framework made up of Perl scripts [21], the freely available Concorde TSP solver [18], and the solver for the maximum weight matching problem based on the $N$-cubed weighted matching algorithm by Gabow [22]. The latter is available through Mathprog at DIMACS (http://www.dimacs.rutgers.edu). In all the considered scenarios steps two through four of CEN were extremely fast, never lasting more than one second. The more time consuming operation was running LPF which however never took more than three hours on an Intel dualcore $1.86 \mathrm{GHz}$ workstation with Linux OS and $16 \mathrm{~GB}$ of RAM. We implemented DIS, RND, ZRND, STATIC, and Max-Min-RE and MinDiff-RE in ns2 [23] with the parameters listed below. Our simulations also take into account all the overhead generated by every possible protocol operation. We used simulations to derive the energy costs for packet communication and route management, as well as the estimates for the $\rho_{q p w}$ parameters that the analytical frameworks for OPT and CEN require.

All our experiments consider realistic parameters of WSNs. There are 400 wireless sensor nodes deployed over a square area of side $L$. Each node transmission radius is $25 \mathrm{~m}$. Each node has an initial energy of $50 \mathrm{~J}$. It generates $512 \mathrm{~B}$ packets at the rate $r=0.5 \mathrm{bps}$ and sends them to the selected sink according to a (hop-based or geographic) shortest path routing. The channel data rate is $250 \mathrm{Kbps}$ (consistent with IEEE 802.15.4 [24]). The transmission power and the receiving power are 0.0144 and $0.0125 \mathrm{~W}$, respectively, according to the specifications of the TR 1000 radio transceiver from $\mathrm{RF}$ Monolithics [25]. We do not consider sleep or idle power consumption. We assume that when not transmitting or receiving packets, node radios are in sleep mode, i.e., consume a negligible fraction of the energy required when the radio is up (we can use an on-board wake-up low-power radio like that described in [26-28], which wakes up a node from sleep mode only when it has a packet to receive).

Sinks are free to move from any of the sites of a $4 \times 4$ and $8 \times 8$ grid to any other site of the grid. They are resource-rich devices, and we assume that sinks can communicate among themselves through high-data rate reliable communications. We vary the number of sinks in the range $[2,8]$. Protocol related parameters are configured as follows. The mandatory time sinks are forced to sojourn at a site in a major configuration $\left(t_{\min }\right)$ varies in the set $\{50$, $100,250\} \mathrm{Ks}$. The threshold $\delta$ that governs a sink movement decision is 0.1 . Finally, nodal energy is partitioned into 40 levels, each being $2.5 \%$ of the initial energy.

To test our solutions we have considered two different nodal deployments: Nodes are either placed on a grid or they are scattered randomly and uniformly throughout the area. In the first case the 400 nodes are placed on a $20 \times$ 20 regular grid within a deployment area of side $L=475 \mathrm{~m}$. The selected transmission radius $(25 \mathrm{~m})$ ensures that every non-border node has exactly four neighbors. In these scenarios the selected routing is shortest path, and each node sends its packets to the closest sink. In the second case, $L$ is set to $300 \mathrm{~m}$. This induces an average network density of 8.15 neighbors per node. The two different deployments allow us to explore the effect of different densities and different energy consumption patterns on our solutions (see Sect. 7.2.2)

The results we present here are obtained by running 100 experiments for each displayed value. (The 95\% confidence interval is depicted in the figures and displayed along with the data on the tables.)

\subsection{Performance evaluation of the proposed heuristics}

\subsubsection{Grid deployment of the sensor nodes}

We start by considering 400 nodes deployed on a $20 \times 20$ grid. The nodal transmission range is $25 \mathrm{~m}$ and the side of the square deployment area is $475 \mathrm{~m}$.

Tables 1 and 2 show the network lifetime induced by the various protocols when varying $t_{\text {min }}$, the number $s$ of sinks, and the number of sink sites $v$. The network lifetime is defined here as the time till energy depletion of the first sensor. Each table entry shows the absolute lifetime (in millions of seconds) and the percentage decrease with respect to OPT (in parentheses). For ZRND we show results with $2^{k}(0<k \leq 3)$ moving sinks because in this case it is possible to divide the deployment area into $2^{k}$ identical square regions.

The centralized heuristic CEN achieves network lifetimes that are remarkably close to the optimum: The gap from OPT is always below $1 \%$. CEN does so well because it starts from the set of (good) configurations that are produced by OPT. CEN uses these same configurations adding intermediate ones and selecting the times the sinks spend in each configuration by solving LPF (Sect. 5) This forces each selected major configuration to last at least $t_{\min }$, and the transient ones the time needed for broadcasting 
Table 1 Lifetime, in millions of seconds (and \% gap from OPT), $4 \times 4$ grid

\begin{tabular}{|c|c|c|c|c|c|c|c|}
\hline$s$ & $\begin{array}{l}t_{\min } \\
(\mathrm{K})\end{array}$ & OPT & CEN & DIS & RND & ZRND & STATIC \\
\hline \multirow[t]{3}{*}{2} & 50 & 46.71 & $46.71(\approx 0)$ & $44.1 \pm .06(5.5)$ & $29 \pm .22(37.9)$ & $30.91 \pm .21(33.8)$ & $11.1(76.2)$ \\
\hline & 100 & 46.71 & $46.71(\approx 0)$ & $43.8 \pm .12(6.2)$ & $28.8 \pm .32(38.3)$ & $30.2 \pm .33(35.3)$ & $11.1(76.2)$ \\
\hline & 250 & 46.71 & $46.7(.02)$ & $43.4 \pm .12(7)$ & $27.4 \pm .44(41.3)$ & $28.5 \pm .41(38.8)$ & $11.1(76.2)$ \\
\hline \multirow[t]{3}{*}{3} & 50 & 61.14 & $61.1(.01)$ & $54 \pm .2(11.6)$ & $38.2 \pm .22(37.5)$ & & $14.8(75.8)$ \\
\hline & 100 & 61.14 & $61.1(.01)$ & $53.3 \pm .22(12.8)$ & $37.6 \pm .33(38.5)$ & & $14.8(75.8)$ \\
\hline & 250 & 61.14 & $61(.07)$ & $52.1 \pm .33(14.7)$ & $35.4 \pm .43(42.1)$ & & $14.8(75.8)$ \\
\hline \multirow[t]{3}{*}{4} & 50 & 75.94 & $75.93(.01)$ & $58.5 \pm .7(22.9)$ & $45.6 \pm .24(39.9)$ & $50.6 \pm .26(33.3)$ & $19.1(74.8)$ \\
\hline & 100 & 75.94 & $75.93(.01)$ & $57.9 \pm .69(23.7)$ & $44.7 \pm .27(41.1)$ & $49.9 \pm .39(34.3)$ & $19.1(74.8)$ \\
\hline & 250 & 75.94 & $75.9(.06)$ & $57.8 \pm .71(23.8)$ & $42.2 \pm .46(44.4)$ & $48.1 \pm .51(36.6)$ & $19.1(74.8)$ \\
\hline \multirow[t]{3}{*}{5} & 50 & 82.42 & $82.41(.01)$ & $62.9 \pm .25(23.6)$ & $50.8 \pm .27(38.3)$ & & $22.3(72.9)$ \\
\hline & 100 & 82.42 & $82.41(.01)$ & $62.4 \pm .32(24.2)$ & $50.2 \pm .33(39)$ & & $22.3(72.9)$ \\
\hline & 250 & 82.42 & $82.41(.17)$ & $61.5 \pm .33(25.3)$ & $48.5 \pm .48(41.1)$ & & $22.3(72.9)$ \\
\hline \multirow[t]{3}{*}{6} & 50 & 84.97 & $84.96(.01)$ & $67.9 \pm .31(20)$ & $55.6 \pm .3(34.5)$ & & $28.8(66.1)$ \\
\hline & 100 & 84.97 & $84.96(.01)$ & $67.5 \pm .28(20.5)$ & $55 \pm .4(35.2)$ & & $28.8(66.1)$ \\
\hline & 250 & 84.97 & $84.96(.01)$ & $67.3 \pm .36(20.7)$ & $53.7 \pm .52(36.8)$ & & $28.8(66.1)$ \\
\hline \multirow[t]{3}{*}{7} & 50 & 87.29 & $87.28(\approx 0)$ & $73.2 \pm .23(16.1)$ & $60.2 \pm .26(31)$ & & $33.7(61.3)$ \\
\hline & 100 & 87.29 & $87.28(\approx 0)$ & $72.9 \pm .28(16.4)$ & $59.7 \pm .34(31.6)$ & & $33.7(61.3)$ \\
\hline & 250 & 87.29 & $87.27(.02)$ & $72.4 \pm .26(17)$ & $58.1 \pm .44(33.4)$ & & $33.7(61.3)$ \\
\hline \multirow[t]{3}{*}{8} & 50 & 88.96 & $88.9(\approx 0)$ & $76.5 \pm .31(14)$ & $63.4 \pm .22(28.7)$ & $59.6 \pm .23(33)$ & $45.2(49.2)$ \\
\hline & 100 & 88.96 & $88.9(\approx 0)$ & $76.1 \pm .32(14.4)$ & $63.1 \pm .31(29)$ & $59.2 \pm .3(33.4)$ & $45.2(49.2)$ \\
\hline & 250 & 88.96 & $88.9(\approx 0)$ & $75.4 \pm .41(15.2)$ & $61.6 \pm .45(30.7)$ & $59 \pm .43(33.6)$ & $45.2(49.2)$ \\
\hline
\end{tabular}

Table 2 Lifetime, in millions of seconds (and \% gap from OPT), $8 \times 8$ grid

\begin{tabular}{|c|c|c|c|c|c|c|c|}
\hline$s$ & $\begin{array}{l}t_{\min } \\
(\mathrm{K})\end{array}$ & OPT & CEN & DIS & RND & ZRND & STATIC \\
\hline \multirow[t]{3}{*}{2} & 50 & 79.51 & $79.49(.02)$ & $68.5 \pm .42(13.8)$ & $39.2 \pm .28(50.7)$ & $47.4 \pm .32(40.3)$ & $14.2(82.1)$ \\
\hline & 100 & 79.51 & $79.49(.02)$ & $67.8 \pm .4(14.7)$ & $39.2 \pm .37(50.7)$ & $46.5 \pm .38(41.4)$ & $14.2(82.1)$ \\
\hline & 250 & 79.51 & $79.2(.4)$ & $64 \pm .75(19.5)$ & $38 \pm .62(52.2)$ & $43.9 \pm .6(45.2)$ & $14.2(82.1)$ \\
\hline \multirow[t]{3}{*}{3} & 50 & 105.9 & $105.9(.03)$ & $90.1 \pm .36(14.9)$ & $50.7 \pm .36(52.1)$ & & $20.8(80.3)$ \\
\hline & 100 & 105.9 & $105.9(.03)$ & $89.3 \pm .32(15.6)$ & $50.7 \pm .54(52.1)$ & & $20.8(80.3)$ \\
\hline & 250 & 105.9 & $105.8(.05)$ & $87.6 \pm .36(17.2)$ & $49.3 \pm .8(53.4)$ & & $20.8(80.3)$ \\
\hline \multirow[t]{3}{*}{4} & 50 & 131.4 & $131.4(.03)$ & $106.4 \pm .33(19)$ & $63.4 \pm .47(51.7)$ & $88 \pm .26(33)$ & $27.7(78.9)$ \\
\hline & 100 & 131.4 & $131.4(.04)$ & $105.7 \pm .43(19.5)$ & $63.6 \pm .5(51.6)$ & $86.9 \pm .41(33.8)$ & $27.7(78.9)$ \\
\hline & 250 & 131.4 & $131.3(.1)$ & $102.5 \pm .64(22)$ & $61.5 \pm .81(53.2)$ & $83.8 \pm .63(36.2)$ & $27.7(78.9)$ \\
\hline \multirow[t]{3}{*}{5} & 50 & 150.1 & $150(.04)$ & $120 \pm .66(20)$ & $75.6 \pm .46(49.6)$ & & $34.3(77.1)$ \\
\hline & 100 & 150.1 & $150(.04)$ & $118.8 \pm .77(20.8)$ & $75.9 \pm .62(49.4)$ & & $34.3(77.1)$ \\
\hline & 250 & 150.1 & $149.9(.1)$ & $117 \pm .51(22)$ & $73.9 \pm .93(50.7)$ & & $34.3(77.1)$ \\
\hline
\end{tabular}

sink activations and deactivations and for the sinks to move. The time the sinks spend in each of these configurations might, in principle, differ substantially from that selected by OPT. However, we notice that the good configurations where OPT sends the sinks for the most time are also those selected by CEN. This is the natural consequence of OPT and CEN being optimization formulations. LPF in CEN optimizes the lifetime and deems it useful to spend long times in those good configurations where traffic is delivered with low energy consumption and the energy toll is balanced among the nodes. These configurations are the major configurations selected by OPT. The additional configurations needed by CEN for transitioning from major configurations are carefully selected to mimic the adjacent major configurations. They also are short compared to major configuration, with a limited impact on the overall 
energy consumption. This explains the near-optimal performance of the centralized heuristic (as mentioned, just $<1 \%$ from OPT).

One may wonder how much OPT and CEN loose making nodes sending their packets to the closest sink rather than to the best one. To answer this question we have run the model by setting this option, and observed that the loss (in lifetime) is quite limited ranging from 3.8 to $8.7 \%$.

The distributed heuristic DIS is aware of the residual energy at the network nodes because of the exchange of information among the sinks. Based on this (approximate) knowledge as well as on the estimate of the nodal data rate and of the energy consumption pattern, DIS can move the sinks to configurations that are expected to improve network lifetime. Traffic and energy-awareness pay off in terms of network lifetime, which is always within $25.3 \%$ from the OPT lifetime. Equally important, the improvement with respect to STATIC is as high as fourfold. This confirms the goodness of exploiting controlled, energyaware sink mobility, especially for multiple sinks.

Sink mobility is advantageous even when uncontrolled, i.e., even when sink movements do not depend on the network state (nodal residual energy, energy consumption patterns, etc.). With RND the random movements of the sinks almost triples the network lifetime with respect to STATIC. ZRND offers similar performance.

We also observe that OPT and the other heuristics induce longer lifetimes when the number of sinks increases. This is because the network traffic is partitioned among a larger set of sinks, sink neighbors receive fewer packets, routes are shorter and the overall energy consumption is lower. However, we notice lifetime improvements which are not linear in the number of sinks. In other words, having two sinks does not double lifetime compared to one sink. Having three sinks does not triple lifetime, and so on. This is evident in Table 1, which shows that deploying 8 sinks only leads to a $17 \%$ improvement in OPT lifetime with respect to scenarios with 4 sinks. Given our set of sink sites, we do not expect that linear improvement is possible. The route length and the overall energy consumption do not halve when we double the number of sinks. In addition, achieving linear improvements would require sinks to be assigned (on average) to configurations which perfectly partition data sources to the sinks. Such perfect configurations are rare, if possible at all. Moreover, if it were possible for sinks to transition only among this kind of node-balanced configurations it would be challenging to obtain good energy balancing. Energy balancing is in fact the consequence of the fine tuning of the time spent by the sinks in different configurations (including unbalanced ones) so that all the nodes relay a similar amount of traffic over time and the energy consumption is minimized.
For all protocols, remarkably higher lifetime is obtained by increasing the number of sink sites $v$. For example, when 5 sinks may visit 64 sites the lifetime is $150.1 \mathrm{Ms}$ for OPT. When restricted to 16 sink sites the lifetime is 82.42 Ms. A higher number of sites allows the protocol to choose among a higher number of configurations. Denser sink sites allow the sinks to drain energy from all the different areas in the network.

We finally observe that both DIS, RND and ZRND produce higher lifetime increases as $t_{\min }$ decreases. The reason is that higher $t_{\mathrm{min}} s$ result in a coarser selection of the times spent in the various configurations, and therefore in a worse energy balancing. In addition, the price to pay for having entered an "energy undesirable" configuration is paid for a longer time.

As a final remark, we observe that our mobility schemes not only improve the average network lifetime, but also consistently perform well in every single run we considered. For instance, in the case with 4 sinks moving among 16 sites and $t_{\min }=50,000 \mathrm{~s}$ the minimum network lifetime for DIS is $54.6 \mathrm{Ms}$, a mere $7 \%$ from the observed average. In the case with 5 sinks moving over 64 sink sites, and $t_{\min }=50,000 \mathrm{~s}$, the average lifetime is $11 \%$ greater than the observed minimum. These two cases are those with the greatest standard deviation of network lifetime for the 16 and 64 scenarios, respectively (in all other scenarios the variance is even smaller).

Beyond achieving improved network lifetime CEN and DIS also result in a more even distribution of the nodes residual energies with respect to RND, ZRND and STATIC (confirming they are able to more evenly distribute the traffic load over different nodes throughout the network lifetime). Ideally, we would like the sinks to coordinately move, changing their positions over time so that the energy is evenly drained from all the areas in the networks. CEN and DIS satisfactorily achieve our goal. This is clearly shown in Fig. 2 which displays the residual energy of the nodes at network lifetime. The figure shows the results of a run on a typical scenario where 5 sinks can select among 64 sites (performance does not significantly change when considering different runs). A lighter color means a lower percentage of residual energy in that area of the network. We observe that CEN results in better energy balancing that DIS, which in turn outperforms RND, ZRND and STATIC. More precisely, at lifetime OPT and CEN show an impressive percentage of nodes with very little energy left, a witness of its good energy drainage balancing property. The fraction of nodes with less than $20 \%(40 \%)$ residual energy at network lifetime is $52.5 \%(80 \%)$. This means that at network lifetime, i.e., when the first sensor dies, many more sensors are also about to cease their operations, which comprises the functioning of the whole network. Specifically, in all considered scenarios we 
observed that from 73 to $96 \%$ of the nodes around all sink sites (the only ones that can relay packets to the sinks) are left with no more than $3 \%$ of their initial energy, which makes communications very limited if not impossible.

In DIS the percentage of nodes with less than $20 \%$ of the initial energy at lifetime is around $36.7 \%$. The percentage of nodes with less than $40 \%$ of the initial energy is $57.2 \%$. These figures reduce to 12.75 and $39.75 \%$ for RNDinduced residual energy, and to 1.75 and $3.5 \%$ for STATIC. In a scenario with 4 moving sinks, these figures for ZRND equates 16 and $52.5 \%$.

The other WSN-relevant metric we have investigated is the end-to-end packet latency (Tables 3, 4). For all protocols, latencies are quite low, never exceeding $200 \mathrm{~ms}$ (the worst case is RND, 16 sinks site, 2 sinks scenario).

Latency performance mostly depends on the average length of the routes traveled by packets. Increasing the number of sinks results in lower route length, and therefore in lower latencies.

When comparing the end-to-end latency experienced by the different schemes, we observe that STATIC achieves the shortest routes almost always because it places the sinks in a well balanced way resulting in short source-tosink routes. CEN and DIS select configurations which spread sinks over different areas in the network, more or less evenly associating nodes to the different sinks. The increase of route length and end-to-end latency of CEN and DIS over STATIC is therefore quite limited, never more than $18 \%$ (for 16 sink sites) or more than $20 \%$ (for 64 sink sites). Both heuristics have comparable performance,

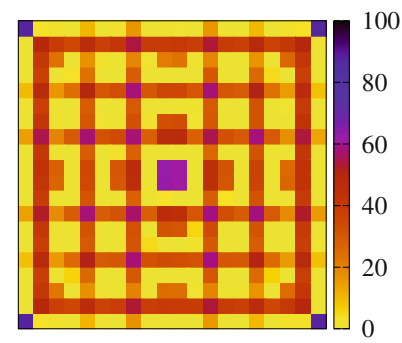

(a) CEN

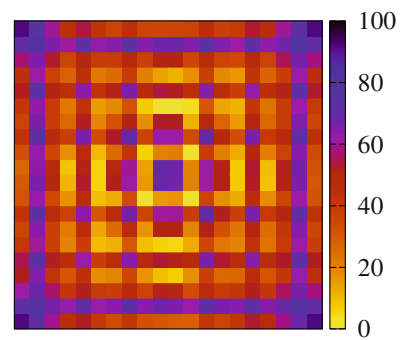

(c) RND

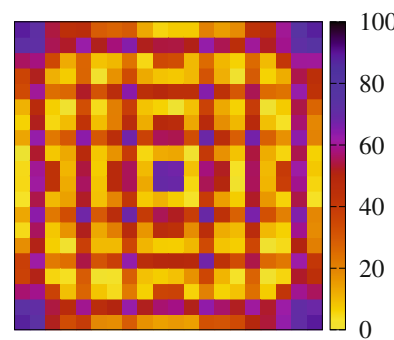

(b) DIS

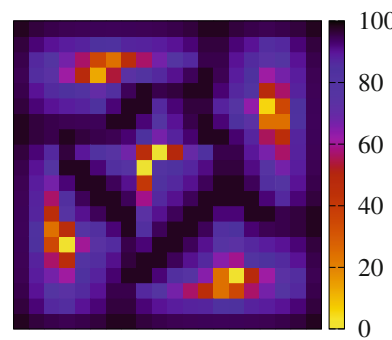

(d) STATIC
Fig. 2 Residual energy at lifetime for CEN (a), DIS (b), RND (c), and STATIC (d), 5 sinks in a $8 \times 8$ grid
Table 3 End-to-end packet latency, in seconds $(4 \times 4$ grid $)$

\begin{tabular}{llllll}
\hline$s$ & CEN & DIS & RND & ZRND & STATIC \\
\hline 2 & .189 & .19 & .2 & .19 & .166 \\
3 & .153 & .15 & .166 & & .129 \\
4 & .129 & .131 & .141 & .13 & .123 \\
5 & .117 & .112 & .124 & & .105 \\
6 & .107 & .103 & .112 & & .105 \\
7 & .097 & .096 & .103 & & .091 \\
8 & .089 & .09 & .094 & .09 & .082 \\
\hline
\end{tabular}

Table 4 End-to-end packet latency, in seconds $(8 \times 8$ grid $)$

\begin{tabular}{llllll}
\hline$s$ & CEN & DIS & RND & ZRND & STATIC \\
\hline 2 & .179 & .177 & .196 & .18 & .154 \\
3 & .139 & .138 & .16 & & .117 \\
4 & .116 & .112 & .137 & .122 & .096 \\
5 & .102 & .102 & .122 & & .087 \\
\hline
\end{tabular}

interchangeably outperforming each other depending on the specific scenario. RND can produce configurations where the sinks are all on one side of the network, thus inducing sensor-to-sink routes that can be long, and thus again longer latencies. In our scenarios we measured an increase of end-to-end latency of up to $12 \%$ with respect to CEN and DIS. ZRND mitigates the unbalanced effects observed for RND, producing latencies that are similar to DIS.

We notice that the above end-to-end packet latency increases/decreases have very limited impact on perceived performance (we are speaking of latencies of few hundred milliseconds) while the increases in network lifetime obtained by energy-aware solutions like DIS (over RND, ZRND and STATIC) make a remarkable difference in terms of the monitoring capability of the network. For instance, in the 16 sink site scenario with 2 sinks (see Table 1), DIS is able to successfully deliver over two million packets during the network lifetime vs. the half a million packets that are delivered in STATIC scenarios. The number of DIS-delivered packets is 3.7 millions and those delivered in STATIC scenario are 2.2 when 8 sinks roam through the network.

Finally, we have run experiments where packets are routed to the sinks through geographic routing (namely, geographic greedy forwarding). We observed trends and values similar to those obtained with shortest path routing. For instance, in scenarios with 8 (4) sinks traveling through 16 sink sites, OPT achieves a lifetime of $89.5 \mathrm{Ms}$ (78.7 Ms) when using geographic routing vs. a lifetime of 88.96 Ms (75.93 Ms) obtained by routing packets via shortest paths. 


\subsubsection{Random and uniform sensor deployment}

In this section we explore the performance of the solutions we proposed when nodes are randomly and uniformly deployed. We consider scenarios where 400 nodes are scattered randomly and uniformly throughout a square area of side $L=300 \mathrm{~m}$. As previously considered, the sensor node transmission radius is $25 \mathrm{~m}$ and the sink sites are deployed in a grid pattern. Packet are routed to the closest sink via shortest paths. We tested 12 different connected topologies, running multiple experiments on each one. The average nodal degree of the considered topologies varies from 7.51 to 8.46 (doubling that of the grid case). We investigated scenarios where 2,3 and 4 sinks roam through the network nodes, sojourning at $v$ sink sites, with $v=16$ and 36.

A different deployment and different (i.e., higher) densities considerably affect nodal energy consumption. In particular, the average energy consumed by a node is considerably reduced. For instance, while the nodal average consumption per second is $4.53 \mathrm{E}-7 \mathrm{~J}$ in the case of grid deployment, when nodes are scattered randomly and uniformly the same metric averages to $2.81 \mathrm{E}-7 \mathrm{~J}$ (with 2 sinks roaming over a $4 \times 4$ sink-site grid). The averages become $3.3 \mathrm{E}-7$ and $2.05 \mathrm{E}-7$, respectively, when we deploy four mobile sinks. Nodal deployment also affects routing, thus inducing different energy consumption patterns. Figure 3 shows the different patterns of energy consumption imposed by routing over different types of nodal deployment when two sinks (red circles) are positioned at two opposite corners of the network. The squares represent sensor nodes. Different shades of gray indicate different energy consumption rates per node $(\mathrm{J} / \mathrm{s})$ : The darker the node the higher the energy consumed per second.

Figure 3 demonstrates that in the grid deployment the nodes that are stressed the most are the immediate neighbors of a sink, and the energy consumption rate is quite regular (Fig. 3(a)). When nodes are scattered randomly and uniformly throughout the area (Fig. 3(b)), critical nodes are bottlenecks in the (non-necessarily radio) vicinity of the sink, and the energy consumption patterns are more irregular. (We have observed similar trends with different configurations.)

Despite these significant differences, our experiments show that the relative trends of the different protocols in terms of the metrics of interest (network lifetime, packet route length and end-to-end latency) are the same whether the sensors are deployed on a grid or randomly and uniformly.

This is clearly indicated by the results shown in Tables 5 and 6 for the lifetime and in Tables 7 and 8 for the end-toend packet latency.
We observe that the values obtained for network lifetime are comparable to those observed for the grid deployment scenario (e.g., Table 1). This is because, despite higher density usually enables better balancing and lower per node energy consumption, moving sinks so that energy consumption is balanced and minimized is much more challenging, given the irregular nodal placement. This motivates a more uneven distribution of nodal residual energy at lifetime in all protocols, including CEN.

For instance, when 4 sinks move in a $6 \times 6$ grid, the fraction of CEN nodes with less than $20 \%(40 \%)$ residual energy at network lifetime is $19.64 \%(32.45 \%)$. The percentage of CEN nodes with less than $60 \%(80 \%)$ of the initial energy is $49.54 \%(73.46 \%)$. In DIS the percentage of nodes with less than $20 \%$ (40\%) of the initial energy at lifetime is $8.86 \%(19.29 \%)$. The percentage of nodes with less than $60 \%(80 \%)$ of the initial energy is $35.39 \%$ $(62.84 \%)$. With the RND heuristic the percentage of nodes with less than $20 \%$ (40\%) of the initial energy at lifetime is $1.03 \%(4.14 \%)$. The percentage of RND nodes with less than $60 \%(80 \%)$ of the initial energy is $14.35 \%(44.8 \%)$. With ZRND, instead, the percentage of nodes with less than $20 \%(40 \%)$ of the initial energy at lifetime is $0.84 \%$ $(2.21 \%)$. The percentage of ZRND nodes with less than $60 \%(80 \%)$ of the initial energy is $8.31 \%(29.37 \%)$. Finally, these figures drop to $1.62,3.81,7.66$ and $16.87 \%$, respectively, for STATIC.

\subsection{Comparative performance evaluation}

We compare the lifetime of our solutions to that induced by the heuristics Max-Min-RE and MinDiff-RE, the best among the three sink mobility schemes presented in [6]. This shows the differences between our approach to controlled and coordinated mobility and schemes that limit sinks to sites along the network perimeter. Previous perimeter-based solutions have effectively improved lifetime for one sink [29].

MinDiff-RE is a centralized scheme where sinks sojourn only at sites at the boundary of the deployment area. The protocol proceeds in rounds. At the beginning of each round, the sinks move to a configuration that minimizes the difference between the maximum and minimum residual energy of the nodes at the end of the round. This difference is computed for all possible configurations. Considering scenarios with $s$ sinks and $v$ sink sites, the number of configurations to check at each round is $\left(\begin{array}{l}v \\ s\end{array}\right)$, which grows exponentially with $s$. This limits the applicability of this method to networks with a limited number of sinks. For instance, the $8 \times 8$ sink site grid scenario considered above has 28 sink sites on the area perimeter, which force 
Fig. 3 Per node energy consumption with different types of deployment

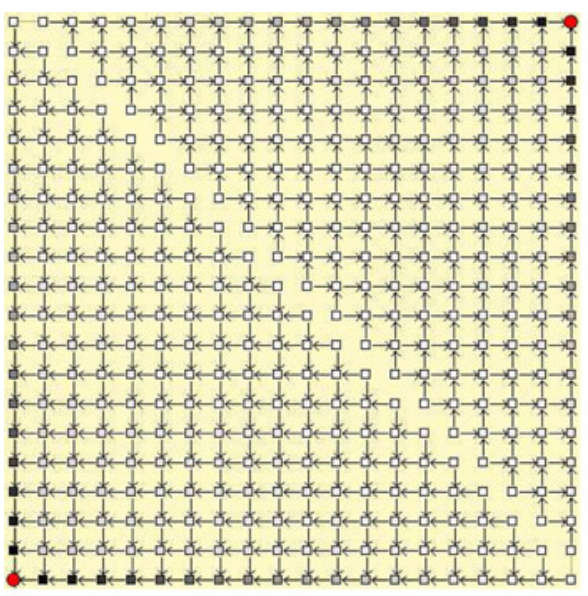

(a) Grid

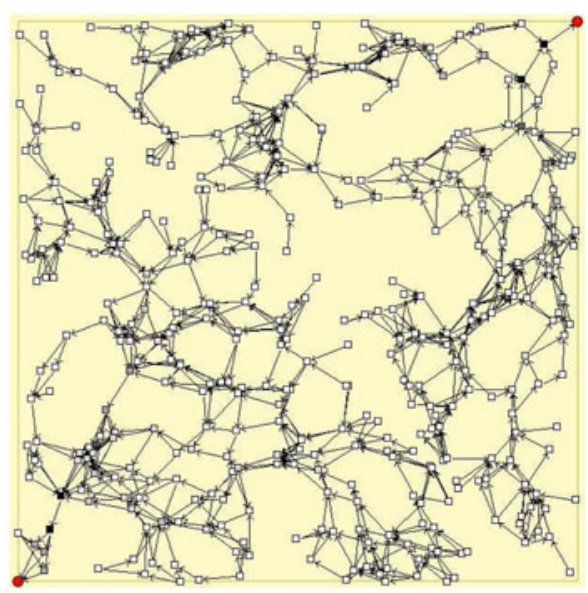

(b) Random deployment

Table 5 Lifetime, in millions of seconds (and \% gap from OPT), $4 \times 4$ grid

\begin{tabular}{rrllllrr}
\hline$s$ & $\begin{array}{l}t_{\min } \\
(\mathrm{K})\end{array}$ & OPT & CEN & DIS & RND & ZRND & STATIC \\
\hline 2 & 50 & $46.9 \pm 2.3$ & $46.9 \pm 2.3(\approx 0)$ & $40.2 \pm 2.4(14.3)$ & $25.8 \pm 1.8(45)$ & $24.3 \pm 2.2(48.2)$ & $15.3(67.3) \pm 1.1$ \\
& 100 & $46.9 \pm 2.3$ & $46.9 \pm 2.3(\approx 0)$ & $40.1 \pm 2.5(14.6)$ & $25.5 \pm 1.6(45.5)$ & $24.1 \pm 2.1(48.6)$ & $15.3(67.3) \pm 1.1$ \\
& 250 & $46.9 \pm 2.3$ & $46.9 \pm 2.3(\approx 0)$ & $39.7 \pm 2.4(15.5)$ & $24.6 \pm 1.5(47.5)$ & $23.4 \pm 1.9(50)$ & $15.3(67.3) \pm 1.1$ \\
3 & 50 & $64.1 \pm 3.1$ & $64.1 \pm 3.1(\approx 0)$ & $53.8 \pm 3.2(16)$ & $32.6 \pm 1.9(49.1)$ & $20.2(68.4) \pm 1.7$ \\
& 100 & $64.1 \pm 3.1$ & $64.1 \pm 3.1(\approx 0)$ & $53.4 \pm 3.2(16.6)$ & $32.5 \pm 1.8(49.3)$ & $20.2(68.4) \pm 1.7$ \\
4 & 250 & $64.1 \pm 3.1$ & $64.1 \pm 3.1(\approx 0)$ & $52.7 \pm 3.3(17.7)$ & $31.6 \pm 1.8(50.7)$ & $20.2(68.4) \pm 1.7$ \\
& 50 & $75.3 \pm 3.4$ & $75.3 \pm 3.4(\approx 0)$ & $63.7 \pm 3.6(15.4)$ & $38.2 \pm 2.3(49.2)$ & $36.7 \pm 3(51)$ & $24.8(67) \pm 1.8$ \\
& 100 & $75.3 \pm 3.4$ & $75.3 \pm 3.4(\approx 0)$ & $63.4 \pm 3.6(15.8)$ & $38.1 \pm 2.3(49.4)$ & $36.8 \pm 3(51)$ & $24.8(67) \pm 1.8$ \\
& 250 & $75.3 \pm 3.4$ & $75.3 \pm 3.4(\approx 0)$ & $62.5 \pm 3.8(16.9)$ & $37.2 \pm 2(50.6)$ & $36 \pm 2.7(52)$ & $24.8(67) \pm 1.8$ \\
\hline
\end{tabular}

Table 6 Lifetime, in millions of seconds (and \% gap from OPT), $6 \times 6$ grid

\begin{tabular}{rrrlllll}
\hline$s$ & $\begin{array}{l}t_{\min } \\
(\mathrm{K})\end{array}$ & OPT & CEN & DIS & ZRND & STATIC \\
\hline 2 & 50 & $56.5 \pm 2.9$ & $56.5 \pm 2.9(.01)$ & $45.1 \pm 2.6(20.1)$ & $25.5 \pm 1.8(54.8)$ & $25.1 \pm 2.6(56)$ & $17(69.9) \pm 1.3$ \\
& 100 & $56.5 \pm 2.9$ & $56.5 \pm 2.9(.01)$ & $45 \pm 2.7(20.3)$ & $25.4 \pm 1.7(55)$ & $24.9 \pm 2.6(55.8)$ & $17(69.9) \pm 1.3$ \\
& 250 & $56.5 \pm 2.9$ & $56.5 \pm 2.9(.06)$ & $44.6 \pm 2.6(21)$ & $24.8 \pm 1.6(56.1)$ & $24.5 \pm 2.4(56.6)$ & $17(69.9) \pm 1.3$ \\
3 & 50 & $82.2 \pm 2.3$ & $82.2 \pm 2.3(.01)$ & $64.1 \pm 3.2(22)$ & $33.1 \pm 2.4(59.7)$ & $37 \pm 2.5(55)$ & $23.6(71.2) \pm 1$ \\
& 100 & $82.2 \pm 2.3$ & $82.2 \pm 2.3(.02)$ & $63.8 \pm 3(22.3)$ & $33.1 \pm 2.3(59.7)$ & $36.9 \pm 2.4(55)$ & $23.6(71.2) \pm 1$ \\
& 250 & $82.2 \pm 2.3$ & $82.2 \pm 2.3(.03)$ & $62.9 \pm 3(23.4)$ & $32.6 \pm 2.2(60.3)$ & $36.5 \pm 2.3(55)$ & $23.6(71.2) \pm 1$ \\
4 & 50 & $105.2 \pm 2.1$ & $105.2 \pm 2.1(.02)$ & $81.9 \pm 2.2(22.1)$ & $40.2 \pm 2.8(61.7)$ & $42.6 \pm 2.7(60)$ & $30.5(71) \pm 1.6$ \\
& 100 & $105.2 \pm 2.1$ & $105.2 \pm 2.1(.02)$ & $81.3 \pm 2.2(22.7)$ & $40.2 \pm 2.8(61.7)$ & $42.8 \pm 2.7(60)$ & $30.5(71) \pm 1.6$ \\
& 250 & $105.2 \pm 2.1$ & $105.2 \pm 2.1(.03)$ & $80.2 \pm 2.2(23.8)$ & $40.1 \pm 2.5(61.8)$ & $42.6 \pm 2.6(60)$ & $30.5(71) \pm 1.6$ \\
\hline
\end{tabular}

thousand of configurations to be checked each round even for $s$ as small as 3 .

Max-Min-RE differs from MinDiff-RE only in the way the new configuration is selected at the beginning of each round. Max-Min-RE chooses the configuration which maximizes the minimum residual energy over all nodes at the end of the round.
Given the scalability problems of the two heuristics, in the following performance comparison we consider only the $4 \times 4$ sink site grid case. Packet are routed to the closest sink via shortest paths. All relevant parameters are set as described as in Sect. 7.2.1 (nodes deployed on a grid). In particular, DIS, RND, Max-Min-RE and MinDiffRE decide whether to move or not, and where, every $t_{\min }$. 
Table 7 End-to-end packet latency, in seconds $(4 \times 4$ grid $)$

\begin{tabular}{|c|c|c|c|c|c|c|}
\hline$s$ & $\begin{array}{l}t_{\min } \\
(\mathrm{K})\end{array}$ & CEN & DIS & RND & ZRND & STATIC \\
\hline \multirow[t]{3}{*}{2} & 50 & $.134 \pm .003$ & $.125 \pm .003$ & $.151 \pm .002$ & $.145 \pm .002$ & $.106 \pm .006$ \\
\hline & 100 & $.133 \pm .003$ & $.125 \pm .003$ & $.15 \pm .002$ & $.145 \pm .001$ & $.106 \pm .006$ \\
\hline & 250 & $.133 \pm .003$ & $.125 \pm .003$ & $.151 \pm .002$ & $.143 \pm .001$ & $.106 \pm .006$ \\
\hline \multirow[t]{3}{*}{3} & 50 & $.108 \pm .003$ & $.104 \pm .002$ & $.125 \pm .001$ & & $.091 \pm .002$ \\
\hline & 100 & $.108 \pm .003$ & $.104 \pm .002$ & $.124 \pm .001$ & & $.091 \pm .002$ \\
\hline & 250 & $.108 \pm .003$ & $.104 \pm .003$ & $.123 \pm .002$ & & $.091 \pm .002$ \\
\hline \multirow[t]{3}{*}{4} & 50 & $.093 \pm .002$ & $.091 \pm .002$ & $.107 \pm .001$ & $.094 \pm .002$ & $.079 \pm .004$ \\
\hline & 100 & $.094 \pm .003$ & $.091 \pm .002$ & $.106 \pm .001$ & $.095 \pm .002$ & $.079 \pm .004$ \\
\hline & 250 & $.093 \pm .003$ & $.091 \pm .002$ & $.106 \pm .001$ & $.095 \pm .002$ & $.079 \pm .004$ \\
\hline
\end{tabular}

Table 8 End-to-end packet latency, in seconds $(6 \times 6$ grid $)$

\begin{tabular}{|c|c|c|c|c|c|c|}
\hline$s$ & $\begin{array}{l}t_{\min } \\
(\mathrm{K})\end{array}$ & CEN & DIS & RND & ZRND & STATIC \\
\hline \multirow[t]{3}{*}{2} & 50 & $.124 \pm .002$ & $.118 \pm .002$ & $.144 \pm .002$ & $.136 \pm .002$ & $.102 \pm .005$ \\
\hline & 100 & $.124 \pm .002$ & $.118 \pm .002$ & $.143 \pm .002$ & $.136 \pm .002$ & $.102 \pm .005$ \\
\hline & 250 & $.124 \pm .002$ & $.118 \pm .002$ & $.142 \pm .001$ & $.137 \pm .002$ & $.102 \pm .005$ \\
\hline \multirow[t]{3}{*}{3} & 50 & $.099 \pm .002$ & $.096 \pm .001$ & $.118 \pm .001$ & $.112 \pm .001$ & $.086 \pm .004$ \\
\hline & 100 & $.099 \pm .002$ & $.096 \pm .001$ & $.118 \pm .001$ & $.112 \pm .001$ & $.086 \pm .004$ \\
\hline & 250 & $.099 \pm .002$ & $.096 \pm .001$ & $.117 \pm .001$ & $.112 \pm .001$ & $.086 \pm .004$ \\
\hline \multirow[t]{3}{*}{4} & 50 & $.085 \pm .001$ & $.083 \pm .001$ & $.102 \pm .001$ & $.09 \pm .001$ & $.076 \pm .002$ \\
\hline & 100 & $.085 \pm .001$ & $.083 \pm .001$ & $.102 \pm .001$ & $.09 \pm .001$ & $.076 \pm .002$ \\
\hline & 250 & $.085 \pm .001$ & $.083 \pm .001$ & $.102 \pm .001$ & $.09 \pm .001$ & $.076 \pm .002$ \\
\hline
\end{tabular}

For fairness reasons, in the scenarios of Max-Min-RE and MinDiff-RE the 16 sink sites are equally spaced along the perimeter of the deployment area.

Figure 4 shows network lifetime results. We observe that the lifetime values induced by Max-Min-RE and MinDiff-RE mobility (the two curves are basically overlapping) are always remarkably worse than those of CEN and DIS. However, Max-Min-RE and MinDiff-RE perform comparably to RND.

The reason for the poor Max-Min-RE and MinDiff-RE performance is limiting the sinks to move only to sites on the boundary of the deployment area, and not within it. As observed in scenarios where the nodes are deployed differently (grid vs. random and uniform), combining configurations that hold the sinks both on the perimeter and inside the deployment area successfully obtains even drainage of energy at the network node. This is a key factor in prolonging network lifetime, which is the objective function that we want to maximize by moving the sinks. Following more geographic/geometric criteria as in Max-Min-RE and MinDiff-RE and limiting through these criteria the movement of the sinks inevitably decreases the possibility of taking advantage of configurations that help in balancing nodal energy consumption.

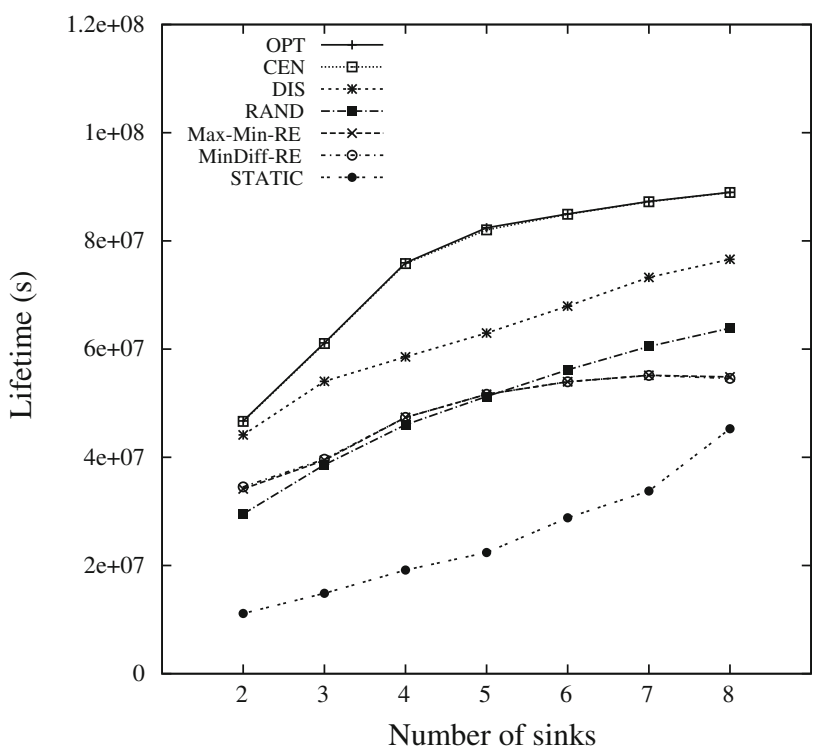

Fig. 4 Network lifetime vs. number of sinks

The values of the residual energy at lifetime confirms this observation.

Finally, keeping the sinks on the perimeter results in longer routes and longer latencies. In order to get to the 
sink closest to its source, a packet has to travel a potentially high number of hops. This results in a higher nodal energy consumption per second (which also explains the results obtained for the network lifetime) and in higher latencies.

\section{Conclusions}

We have shown that controlled and coordinated mobility of multiple sinks effectively improves the lifetime of a WSN. By defining a mathematical model that takes into account realistic parameters, we have provided a provable upper bound on the lifetime of WSNs of realistic scale (OPT). We also defined a centralized heuristic (CEN) for controlled sink mobility. It determines routes and sojourn times for multiple sinks that depend on the network state and produces network lifetimes remarkably close to the upper bound. We then defined a distributed protocol (DIS) for controlled and coordinated sink movements based on the expected lifetime improvements produced by a sink moving to a new site. Comparative performance evaluation among OPT, CEN, DIS, uncontrolled, random mobility, optimal static sink placement and previously proposed solutions for moving multiple sinks shows that coordinating and controlling the mobility of the sinks is always advantageous, yielding remarkable lifetime improvements.

Acknowledgments The authors are grateful to Bob Carr of Sandia National Laboratories for useful comments on the topics of this paper. This work was partially supported by NSF grant \#0738720 and by the FP7 EU project "SENSEI, Integrating the Physical with the Digital World of the Network of the Future," Grant Agreement Number 215923, http://www.ict-sensei.org. Sandia is a multipurpose laboratory operated by Sandia Corporation, a Lockheed-Martin Company, for the United States Department of Energy's National Nuclear Security Administration under contract DE-AC04-94AL85000.

\section{Appendix: Computing transient configurations}

A transition between configuration $c_{i} \in C$ to configuration $c_{j} \in C$ is legal if $\left|c_{j}-c_{i}\right| \leq s-\left|c_{i}\right|$. That is, in a legal transition, the number of sites that receive new active sinks in the new configuration does not exceed the number of inactive sinks in the preceding configuration. The inactive sinks can move to the new sites during the time configuration $c_{i}$ holds. In addition we require that at least one sink is active at all times.

We now show that we can transition between any pair of major configurations using at most two transient configurations.

Theorem 1 At most two transient configurations suffice to move from any major configuration to the following one.
Proof Suppose we wish to transition from configuration $c_{i} \in C$ to $c_{j} \in C$ where $c_{i} \neq c_{j}$. There are only three possible cases for the number of transient configurations between $c_{i}$ and $c_{j}$ :

1. $c_{i} \subset c_{j}$ or $c_{j} \subset c_{i}$. In this case, there are no transient configurations necessary since moving from $c_{i}$ to $c_{j}$ or vice versa satisfies our legal-transition criteria. If $c_{i} \subset c_{j}$, then $\left|c_{j}-c_{i}\right|=\left|c_{j}\right|-\left|c_{i}\right| \leq s-\left|c_{i}\right|$, since $\left|c_{j}\right| \leq s$. If $c_{j} \subset c_{i},\left|c_{j}-c_{i}\right|=0 \leq s-\left|c_{i}\right|$, since $\left|c_{i}\right| \leq s$.

2. $\quad c_{i} \not \subset c_{j}$ and $c_{j} \not \subset c_{i}$, and either $c_{i} \cap c_{j} \neq \emptyset$ or $\left|c_{i}\right|<s$ or $\left|c_{j}\right|<s$. In this case, one transient configuration suffices. If $c_{i} \cap c_{j} \neq \emptyset$, this intersection is a legal transient configuration $c_{i}^{a}$. From the argument for the previous case, we can move directly from configuration $c_{i}$ to $c_{i}^{a}=c_{i} \cap c_{j}$ because $c_{i} \cap c_{j} \subset c_{i}$. Similarly we can move directly from configuration $c_{i}^{a}$ to $c_{j}$ because $c_{i}^{a}=c_{i} \cap c_{j} \subset c_{j}$. If $c_{i} \cap c_{j}=\emptyset$ but $\left|c_{i}\right|<s$, then let $c_{i}^{a}$ be any element of $c_{j}$. Moving from $c_{i}^{a}$ to $c_{j}$ is legal because $c_{i}^{a} \subset c_{j}$. Since $\left|c_{i}\right|<s$ and configuration sizes are integers, we have $s-\left|c_{i}\right| \geq 1$. Moving from $c_{i}$ to $c_{i}^{a}$ is legal because $\left|c_{i}^{a}-c_{i}\right|=1 \leq s-\left|c_{i}\right|$. A similar argument holds if $\left|c_{j}\right|<s$.

3. $c_{i} \not \subset c_{j}$ and $c_{j} \not \subset c_{i}, c_{i} \cap c_{j}=\emptyset$ and $\left|c_{i}\right|=\left|c_{j}\right|=s$. This covers all the remaining cases. Two transient configurations suffice. Let $c_{i}^{a}$ be any nonempty proper subset of $c_{i}$ and let $c_{i}^{b}$ be any nonempty proper subset of $c_{j}$ such that $\left|c_{i}^{a}\right|+\left|c_{i}^{b}\right|=s$. We can move directly from configuration $c_{i}$ to $c_{i}^{a}$ because $c_{i}^{a} \subset c_{i}$. Similarly, we can move directly from $c_{i}^{b}$ to $c_{j}$ because $c_{i}^{b} \subset c_{j}$. We can move directly from $c_{i}^{a}$ to $c_{i}^{b}$ because $\left|c_{i}^{a}-c_{i}^{b}\right|=\left|c_{i}^{a}\right|=$ $s-\left|c_{i}^{b}\right|$. The first equality follows because $c_{i} \cap c_{j}=\emptyset$ and $c_{i}^{a} \subset c_{i}$ and $c_{i}^{b} \subset c_{j}$. Thus $c_{i}^{a} \cap c_{i}^{b}=\emptyset$. The second equality follows by construction.

In the part of the proof of Theorem 1 concerning the case with one transient configuration, there were three separate sufficient conditions: $c_{i} \cap c_{i+1} \neq \emptyset$ or $\left|c_{i}\right|<s$ or $\left|c_{i+1}\right|<s$. Our argument that one transient configuration suffices used three different constructions for that transient configuration, one for each condition. However, these conditions and constructions are independent and fully compatible. We can combine all the pieces to get a transient configuration with a maximal number of active sinks. This heuristically minimizes the total energy the transient configuration requires per unit time.

A maximal legal configuration $c_{i}^{a}$ for this case contains the sites in $c_{i} \cap c_{i+1}$ plus $s-\left|c_{i}\right|$ elements of $c_{i+1}-c_{i}$ and $s-\left|c_{i+1}\right|$ elements of $c_{i}-c_{i+1}$. For example, suppose $c_{i}=$ $\left\{v_{1}, v_{2}, v_{3}\right\}$ and $c_{i+1}=\left\{v_{3}, v_{4}, v_{5}\right\}$, with $s=4$. Then a possible single transient state is $c_{i}^{a}=\left\{v_{2}, v_{3}, v_{4}\right\}$. At the 
start of the transition, the one spare sink, not active during configuration $c_{i}$, has finished traveling to location $v_{4}$. To move to configuration $c_{i}^{a}$, the sink waiting at site $v_{4}$ activates while the sink at location $v_{1}$ deactivates and begins moving to location $v_{5}$. When the traveling sink arrives at $v_{5}$, it activates, while the (now spare) sink at location $v_{2}$ deactivates to put the system in configuration $c_{i+1}$.

The specific way we construct a single transient configuration between $c_{i}$ and $c_{i+1}$ in step 4 of the centralized heuristic is to include the entire intersection $c_{i} \cap c_{i+1}$ plus $s-\left|c_{i}\right|$ randomly selected sites from $c_{i+1}-c_{i}$ and $s-$ $\left|c_{i+1}\right|$ randomly selected sites from $c_{i}-c_{i+1}$. There may be some advantage to making more clever choices for the spare sinks, but this case rarely arises. In practice, if a configuration does not use all $s$ sinks, it is almost always a pure subset of at least one other configuration.

If configurations $c_{i}$ and $c_{i+1}$ have no site in common and both have size $s$, we compute two transient configurations as in the proof of Theorem 1. That is, we will choose $c_{i}^{a}$ a nonempty proper subset of $c_{i}$ and $c_{i}^{b}$ a nonempty proper subset of $c_{i+1}$ such that $\left|c_{i}^{a}\right|+\left|c_{i}^{b}\right|=s$. For example, in Fig. 1, both configurations $c_{i}$ and $c_{i+1}$ use all 6 possible sinks. $c_{i}^{a}=\left\{v_{1}, v_{3}, v_{5}\right\}$ and $c_{i}^{b}=\left\{v_{7}, v_{9}, v_{11}\right\}$. To make the transition, the sinks at sites in $c_{i}-c_{i}^{a}$ move first to locations in $c_{i}^{b}$ while those in $c_{i}^{a}$ stay where they are to receive data. Then the sinks in $c_{i}^{a}$ move to their final positions while those in $c_{i}^{b}$ receive data. There are many possible ways to choose $c_{i}^{a}$ as a subset of $c_{i}$ and $c_{i}^{b}$ as a suitablysized subset of $c_{i+1}$. We now describe one way to pick these sets and argue why heuristically we might expect it to perform well compared to using an arbitrary pair of sets that satisfy our constraints.

To create the transient configurations between major configurations $c_{i}$ and $c_{i+1}$, we first consider configuration $c_{i}$. We estimate the distance between each pair of sink sites in $c_{i}$. We then construct a complete graph that has a node for each site in $c_{i}$, and edges weighted by this pairwise distance estimate. We find a minimum-weight maximumcardinality matching in this graph. We then pick an element from each matched pair arbitrarily to form the set of sink sites $c_{i}^{a}$. For instance, in the upper left corner of Fig. 1, lines show the matching for configuration $c_{i}$. The configuration $c_{i}^{a}=\left\{v_{1}, v_{3}, v_{5}\right\}$ is the unboxed element of each matched pair. The set of sink sites to move first is $M_{1}=c_{i}-c_{i}^{a}=\left\{v_{2}, v_{4}, v_{6}\right\}$. To move from $c_{i}$ to $c_{i}^{a}$, the sinks at sites in $M_{1}$ deactivate and begin to move while those in $c_{i}^{a}$ stay to receive traffic. We hope that if sites $v_{i}$ and $v_{j}$ are matched and a sink at site $v_{i}$ deactivates to move, the nodes sending to $v_{i}$ will be redirected to the nearby site $v_{j}$, maintaining an energy consumption pattern among nodes that is similar to that of configuration $c_{i}$. We then compute a similar matching in $c_{i+1}$ and use that to pick the new locations $M_{2}$ for the sinks from sites in $M_{1}$ to move to. Thus $c_{i}^{b}=M_{2}$. We hope $c_{i}^{b}$ approximates the energy balance of configuration $c_{i+1}$.

\section{References}

1. Yick, J., Mukherjee, B., \& Ghosal, D. (2008). Wireless sensor network survey. Computer Networks, 52(12), 2292-2330.

2. Chang, J.-H., \& Tassiulas, L. (2000). Energy conserving routing in wireless ad hoc networks. In Proceedings of IEEE infocom 2000, Tel Aviv, Israel, Vol. 1, pp. 22-31, March 26-30.

3. Cheng, Z., Perillo, M., \& Heinzelman, W. B. (2008). General network lifetime and cost models for evaluating sensor network deployment strategies. IEEE Transactions on Mobile Computing, 7(4), 484-497.

4. Mak, N. H., \& Seah, W. K. G. (2009). How long is the lifetime of a wireless sensor network? In Proceedings of the IEEE 23rd international conference on advanced information networking and applications, AINA 2009, Bradford, UK, pp. 763-770, May 26-29.

5. Basagni, S., Carosi, A., Melachrinoudis, E., Petrioli, C., Wang, Z. M. (2008). Controlled sink mobility for prolonging wireless sensor networks lifetime. ACM/Springer Wireless Networks, 14(6), 831-858.

6. Azad, A. P., \& Chockalingam, A. (2006). Mobile base station placement and energy aware routing in wireless sensor networks. In Proceeding of the IEEE wireless communications and networking conference, WCNC 2006, Las Vegas, NV, Vol. 1, pp. 264-269, April 3-6.

7. Gandham, S. R., Dawande, M., Prakash, R., \& Venkatesan, S. (2003). Energy efficient schemes for wireless sensor networks with multiple mobile base stations. In Proceedings of IEEE globecom 2003, San Francisco, CA, Vol. 1, pp. 377-381, December $1-5$.

8. Ren, B., Ma, J., \& Chen, C. (2006). The hybrid mobile wireless sensor networks for data gathering. In Proceedings of the ACM international conference on wireless communications and mobile computing, IWCMC 2006, Vancouver, BC, pp. 1085-1090, July 3-6.

9. Chen, C., Ma, J., \& Yu, K. (2006). Designing energy-efficient sensor networks with mobile sinks. In Proceedings of the 1st workshop on world-sensor-web: Mobile device centric sensory networks and applications, WSW 2006, Boulder, CO, October 31.

10. Chatzigiannakis, I., Kinalis, A., Nikoletseas, S., \& Rolim, J. (2007). Fast and energy efficient sensor data collection by multiple mobile sinks. In Proceedings of the 5th ACM international workshop on mobility management and wireless access, MobiWac 2007, Chania, Crete Island, pp. 25-32, October 22. ACM.

11. Hillier, F. S., \& Lieberman, G. J. (2005). Introduction to operations research (8th ed.). Boston, MA: McGraw Hill.

12. Chandru, V., \& Rao, M. R. (1998). Linear programming. In M. Atallah (Eds.), Algorithms and theory of computation handbook. Boca Raton, FL: CRC Press.

13. Lovász, L., Grötschel, M., \& Schrijver, A. (1981). The ellipsoid method and its consequences in combinatorial optimization. Combinatorica, 1, 169-197.

14. Motwani, R., O'Callaghan, L., \& Zhu, A. (2007). Asymptotic polynomial-time approximation schemes. In G. Gonzalez (Eds.), Handbook of approximation algorithms and metaheuristics. Boca Raton, FL: CRC Press.

15. Schrijver, A. (1998). Theory of linear and integer programming. New York: Wiley. 
16. Revelle, C., \& Swain, R. (1970). Central facilities location. Geographical Analysis, 2, 30-42.

17. Berry, J., Hart, W., Phillips, C., Uber, J., \& Watson, J.-P. (2006). Sensor placement in municipal water networks with temporal integer programming models. Journal of Water Resources Planning and Management, 132, 218-224.

18. Concorde TSP Solver. (2005). http://www.tsp.gatech.edu/ concorde.html.

19. Christofides, N. (1976). Worst-case analysis of a new heuristic for the travelling salesman problem. Technical report 388, Carnegie-Mellon University, Graduate School of Industrial Administration.

20. ILOG, CPLEX Home Page. (2004). http://www.ilog.com/ products/cplex/.

21. Deitel, H. M., Deitel, P. J., Nieto, T. R., \& McPhie, D. C. (2000). Perl how to program. Englewood Cliffs, NJ: Prentice Hall.

22. Gabow, H. N. (1973). Implementation of algorithms for maximum matching on non-bipartite graphs. $\mathrm{PhD}$ thesis, Stanford University, Department of Computer Science, Palo Alto, CA.

23. The VINT Project. (2002). The ns manual. http://www.isi.edu/ nsnam $/ \mathrm{ns} /$.

24. IEEE Standard for Information Technology. (2003). Part 15.4: Wireless medium access control (MAC) and physical layer (PHY). Specifications for low-rate wireless personal area networks (LR-WPANs).

25. ASH Transceiver Designer's Guide. (2004). http://www.rfm.com, May 19, 2004.

26. Gu, L., \& Stankovic, J. A. (2005). Radio-triggered wake-up for wireless sensor networks. Real-Time Systems, 29(2-3), 157-182.

27. Kolinko, P., \& Larson, L. E. (2007). Passive RF receiver design for wireless sensor networks. In Proceeding of the IEEE/MTT-S international microwave symposium, IMS 2007, Honolulu, HI, pp. 567-570, June 3-8.

28. Lu, G., De, D., Xu, M., Song, W.-Z., \& Shirazi, B. (2009). A wake-on sensor network. In Proceedings of the 7th ACM conference on embedded networked sensor systems, SenSys 2009, pp. 341-342, November 4-6.

29. Luo, J., \& Hubaux, J.-P. (2005). Joint mobility and routing for lifetime elongation in wireless sensor networks. In Proceedings of IEEE infocom 2005, Miami, FL, Vol. 3, pp. 1735-1746, March $13-17$.

\section{Author Biographies}

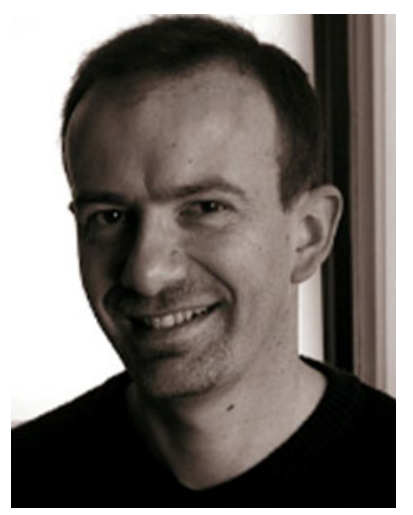

Stefano Basagni holds a Ph.D. in electrical engineering from the University of Texas at Dallas (December 2001) and a Ph.D. in computer science from the University of Milano, Italy (May 1998). He received his B.Sc. degree in computer science from the University of Pisa, Italy, in 1991. Since Winter 2002 he is on faculty at the Department of Electrical and Computer Engineering at Northeastern University, in Boston, MA, where he is currently associate professor. From August 2000 to January 2002 he was assistant professor of computer science at the Department of Computer Science of the Erik Jonsson School of Engineering and Computer Science, The University of Texas at Dallas. Dr. Basagni's current research interests concern research and implementation aspects of mobile networks and wireless communications systems, Bluetooth and sensor networking, definition and performance evaluation of network protocols and theoretical and practical aspects of distributed algorithms. Dr. Basagni has published over five dozens of refereed technical papers and book chapters. He is also co-editor of two books. Dr. Basagni served as a guest editor of the special issue of the Journal on Special Topics in Mobile Networking and Applications (MONET) on Multipoint Communication in Wireless Mobile Networks, of the special issue on mobile ad hoc networks of the Wiley's Interscience's Wireless Communications \& Mobile Networks journal, of the special issue of the Elsevier's journal Ad Hoc Networks on advances in ad hoc and sensor networking, and of the special issue of the Elsevier's journal Algorithmica on algorithmic aspects of mobile computing and communications. Dr. Basagni serves as a member of the editorial board and of the technical program committee of ACM and IEEE journals and international conferences. He is a senior member of the ACM (including the ACM SIGMOBILE), a senior member of the IEEE (Computer and Communication societies), a member of ASEE (American Society for Engineering Education) and of CUR (Council on Undergraduate Research).

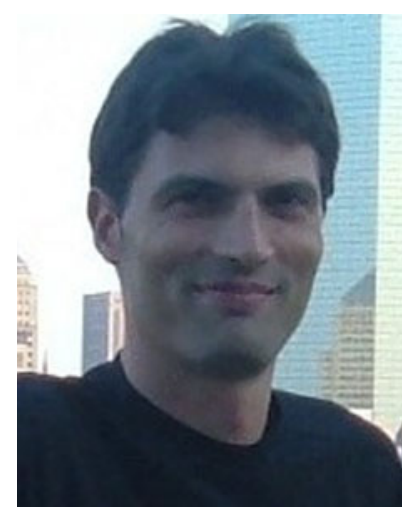

Alessio Carosi received the $\mathrm{Ph} . \mathrm{D}$. in Computer Science in February 2009 from Rome University "La Sapienza." $\mathrm{He}$ is currently with the R\&D Department of Cambridge Silicon Radio P.L.C. (Cambridge, UK), working on issues of coexistence of IEEE 802.11 and Bluetooth technologies. His current research interests include protocols for ad hoc networks and mobile sensor networks. Mr. Carosi is co-author of around ten refereed technical papers and book chapters.

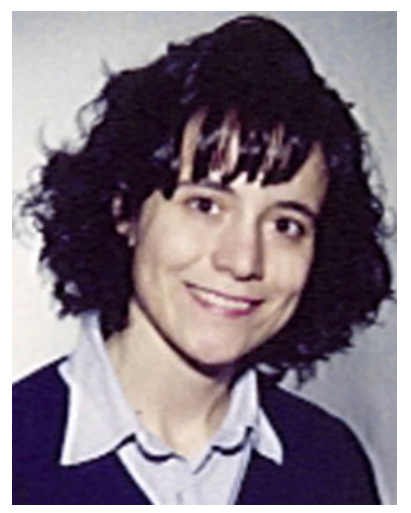

Chiara Petrioli is Associate Professor at the Computer Science Department at Rome University "La Sapienza." She has received the Laurea Degree in Computer Science with the highest honors and the Ph.D. in Computer Engineering from Rome University "La Sapienza,"Italy, in 1993 and 1998, respectively. Prior to her current appointment she was research associate at Politecnico di Milano and was working with the Italian Space agency (ASI) and Alenia Spazio. Dr. Petrioli's work focuses on the design, evaluation and test of protocol stacks for wireless systems, where she contributed over six dozens papers published in prominent international journals and conferences. Her current research interests include ad hoc and sensor networks, personal area networks, cognitive radio networks, underwater acoustic sensor networks, and energy constrained communications protocols. Dr. Petrioli was guest editor of the special issue on "Energy-conserving protocols in wireless Networks" of the ACM/Springer Journal on Special Topics in Mobile Networking and Applications (ACM MONET) and is associate editor of IEEE Transactions on Mobile Computing, the ACM/Springer Wireless Networks journal, the Wiley InterScience Wireless Communications \& Mobile Computing journal and the Elsevier Ad Hoc Networks journal. She has served in the organizing committee and technical program committee of several leading conferences in the 
area of networking and mobile computing including ACM Mobicom, ACM Mobihoc, IEEE INFOCOM, IEEE SECON, IEEE ICC, IEEE Globecom. She is member of the steering committee of ACM Sensys and of the international conference on Mobile and Ubiquitous Systems: Networking and Services (Mobiquitous). She also serves as member of the ACM SIGMOBILE executive committee. Dr. Petrioli was a Fulbright scholar, she is a senior member of IEEE and a member of ACM.

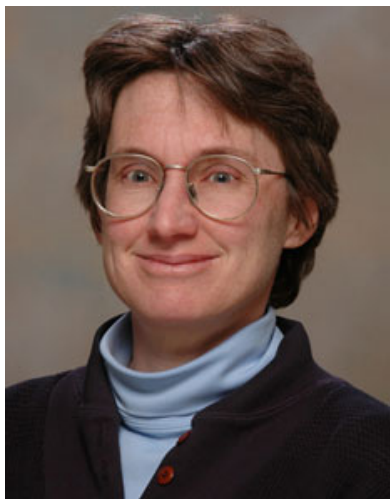

Cynthia A. Phillips received a $\mathrm{PhD}$ in computer science from MIT in 1990. She received a B.A. in applied mathematics from Harvard University in 1983. She is currently a Senior Scientist in the Discrete Mathematics \& Complex Systems Department at Sandia National Laboratories. She joined Sandia National Laboratories in 1990 as a senior member of the technical staff in 1990. Dr. Phillips' current research areas include combinatorial optimization, algorithm design and analysis, experimental algorithmics, and parallel computation. She is one of three main developers of the PICO massively parallel integer programming code. Her current applications areas include network and infrastructure surety, graph algorithms, scheduling for quantum computer architecture, and management of wireless sensor networks. Dr. Phillips has published over 40 refereed technical papers. She has served as a program committee member, sometimes chair, of many international program committees including the International Workshop on Foundations of Mobile Computing (2000-2008, co-chair 2004). She is the program director for the SIAM special Special Interest group on supercomputing and was an officer for the ACM Symposium on Parallelism in Algorithms and Architecture 2000-2004. She recieved an R\&D 100 award in 2006 and in 2008 was a member of a finalist team for the Edeman Award for excellence in operations research practice. 